\title{
The phylogeny of the Schistosomatidae based on three genes with emphasis on the interrelationships of Schistosoma Weinland, 1858
}

\author{
A. E. LOCKYER ${ }^{1}$, P. D. OLSON${ }^{1}$, P. ØSTERGAARD ${ }^{1}$, D. ROLLINSON ${ }^{1}$, D. A. JOHNSTON ${ }^{1}$, \\ S. W. ATTWOOD ${ }^{1}$, V. R. SOUTHGATE ${ }^{1}$, P. HORAK ${ }^{2}$, S. D. SNYDER ${ }^{3}$, \\ T. H. LE ${ }^{4}$, T. AGATSUMA ${ }^{5}$, D. P. McMANUS ${ }^{6}$, A. C. CARMICHAEL ${ }^{7}$, \\ S. NAEM ${ }^{8}$ and D. T. J. LITTLEWOOD ${ }^{1 *}$
}

${ }^{1}$ Department of Zoology, The Natural History Museum, Cromwell Road, London SW7 5BD, UK

${ }^{2}$ Department of Parasitology and Hydrobiology, Charles University, Vinicna 7, CZ-128 44 Prague 2, Czech Republic

${ }^{3}$ Department of Biology, University of Nebraska at Omaha, 6001 Dodge Street, Omaha, NE 68182-0040, USA

${ }^{4}$ Immunology Department, Institute of Biotechnology of Vietnam, 18. Hoang Quoc Viet Road, Cau Giay District, Hanoi, Vietnam

${ }^{5}$ Department of Environmental Health Science, Faculty of Medicine, Kochi Medical School, Oko, Nankoku City, Kochi 783-8505, Fapan

${ }^{6}$ Molecular Parasitology Laboratory, The Queensland Institute of Medical Research, 300 Herston Road, Brisbane, Q4029, Australia

${ }^{7}$ University of California Botanical Garden, University of California, 200 Centennial Drive, Berkeley, CA 94720, USA

${ }^{8}$ Nazloo Campus, Department of Pathobiology, Faculty of Veterinary Medicine, Urmia, Iran

(Received 12 September 2002; revised 26 October 2002; accepted 26 October 2002)

\section{S U M MAR Y}

Schistosomes are digenean flukes, parasitic of birds, mammals and crocodiles. The family Schistosomatidae contains species of considerable medical and veterinary importance, which cause the disease schistosomiasis. Previous studies, both morphological and molecular, which have provided a good deal of information on the phylogenetics of this group, have been limited in the number of species investigated or the type or extent of molecular data used. This paper presents the most comprehensive phylogeny to date, based on the sequences of 3 genes, complete ribosomal small subunit rRNA and large ribosomal subunit rRNA, and mitochondrial cytochrome oxidase 1, sequenced from 30 taxa including at least 1 representative from 10 of the 13 known genera of the Schistosomatidae and 17 of the 20 recognized Schistosoma species. The phylogeny is examined using morphological characters, intermediate and definitive host associations and biogeography. Theories as to the origins and spread of Schistosoma are also explored. The principal findings are that Ornithobilharzia and Austrobilharzia form a sister group to the Schistosoma; mammalian schistosomes appear paraphyletic and 2 Trichobilharzia species, T. ocellata and T. szidati, seem to be synonymous. The position of Orientobilharzia within the Schistosoma is confirmed, as is an Asian origin for the Schistosoma, followed by subsequent dispersal through India and Africa.

Key words: interrelationships, character analysis, biogeography, host-parasite associations, Digenea.

\section{INTRODUCTION}

The Schistosomatidae are digenean flukes that parasitize birds, mammals and crocodiles and use gastropod intermediate hosts. Schistosomatids are primarily associated with freshwater habitats and are found in all temperate and tropical regions of the world. There are 14 recognized genera and approximately 100 species of schistosomes (Khalil, 2002) including a number of species of medical and veterinary importance. As the causative agents of schistosomiasis, human schistosomes rank amongst the most important of all metazoan parasites, affecting over 220 million people (WHO, 2001). Other

* Corresponding author: Department of Zoology, The Natural History Museum, Cromwell Road, London SW7 5BD, UK. Tel: +44207942 5742. Fax: +44 207942 5151.E-mail: T.Littlewood@nhm.ac.uk schistosomatids, such as the avian flukes Trichobilharzia, also have implications for human health, as the release of their cercariae can cause severe cercarial dermatitis (e.g. Horak \& Kolarova, 2001; Horak, Kolarova \& Adema, 2002). A sound framework for the taxonomy of schistosomes may provide a better understanding of the origins, radiation and evolution of schistosomes. The elucidation of the history, present distribution, and the possible future spread of schistosomes, had implications for controlling the diseases they cause. General descriptions of the family and taxonomic histories can be found in Farley (1971) and Gibson, Jones \& Bray (2002).

Within the Schistosomatidae, it is the genus Schistosoma that contains species that parasitize man. Traditional groupings of Schistosoma species, based primarily on egg morphology, intermediate host specificity and biogeography, divided the genus into 
4 groups, represented by the species $S$. mansoni, $S$. haematobium, S. indicum and S. japonicum (Rollinson \& Southgate, 1987). S. mansoni, which causes human intestinal schistosomiasis, has lateral spined eggs and uses Biomphalaria snails as intermediate hosts. S. mansoni is widespread in Africa and is also present in South America and the Caribbean. Other members of this group include $S$. rodhaini, a rodent parasite and also 2 parasites of the hippopotamus, S. edwardiense and S. hippopotami. S. haematobium causes urinary schistosomiasis in man and uses snails of the genus Bulinus as its intermediate hosts. This species has terminal spined eggs. Most of the other African species fall into this group, such as $S$. intercalatum, which also infects man and several species that infect mainly cattle and sheep, including $S$. bovis, S. mattheei and S. curassoni. It has been estimated that at least 165 million cattle worldwide are infected with schistosomiasis (de Bont \& Vercruysse, 1997). The third group includes $S$. japonicum, which has a rounded, minutely spined egg. S. japonicum is widespread throughout East Asia, although eradicated from Japan by extensive control programmes. Other Asian species in this group are $S$. sinensium, $S$. mekongi, $S$. malayensis and a fourth, recently described, species S.ovuncatum (Attwood et al. 2002a). Both S. mekongi and $S$. japonicum are human pathogens. The $S$. indicum group contains the Indian species $S$. incognitum, $S$. spindale and $S$. nasale, in addition to $S$. indicum. None of these infect man, and they have a variety of egg morphologies. These species have been grouped together for convenience, as much on the basis that they do not fit with the S. mansoni, S. haematobium and $S$.japonicum groups, as that they are all found in India and parts of S.E. Asia (Rollinson \& Southgate, 1987). Indeed, Agatsuma et al. (2002) suggested the group may not be monophyletic. Those species which infect man do not fall into a single species group, indicating that they are not closely related and do not share the same morphological features, intermediate host or geographical ranges. Rather, they individually share features with other species that are not infective to man, and this indicates that there have been independent lateral transfers into man from other hosts (Combes, 1990).

\section{Taxonomy and systematics}

Carmichael (1984) carried out a cladistic analysis of the Schistosomatidae and produced a comprehensive review with a phylogeny based on 24 morphological characters scored for 14 genera. Morand \& MüllerGraf (2000) re-analysed these data using modern computational methods, recoded as 37 characters (Carmichael's thesis included a number of multistate characters). The preferred tree presented by Carmichael was not the most parsimonious as found by a cladistic analysis performed by Morand \& Müller-Graf (2000) using the same characters which provided a more resolved solution (Fig. 1A). There have been a number of molecular attempts to infer schistosomatid phylogenies, with particular emphasis on resolving the interrelationships of species of the medically important genus Schistosoma. Rollinson et al. (1997) reviewed some of the earliest studies based on mitochondrial and nuclear ribosomal gene sequences (Després et al. 1992; Johnston, Kane \& Rollinson, 1993; Littlewood \& Johnston, 1995), RAPDs (Barral et al. 1993; Kaukas et al. 1994) and RFLPs of mitochondrial DNA (Després, ImbertEstablet \& Monnerot, 1993). The majority of these studies involved only a few exemplar taxa and concentrated on Schistosoma. Snyder \& Loker (2000) broadened the approach to the Schistosomatidae and used large subunit ribosomal DNA (lsrDNA). Using 12 ingroup taxa (representing 10 genera) and 2 outgroups, and sequencing about a kilobase of lsrDNA encompassing variable domains D1-D2, their solution differed fundamentally from analyses based on morphology by Morand \& Müller-Graf (2000) (Fig. 1). With lsrDNA sequence data, Orientobilharzia and Schistosoma formed a monophyletic (mammalian) clade and the other schistosomatid taxa formed a primarily avian clade, not seen in the morphology tree. The interrelationships of the remaining bird and mammal schistosomes are the same in both analyses, recognizing 3 clades comprising: Schistosomatium and Heterobilharzia; Dendritobilharzia, Gigantobilharzia, Trichobilharzia and Bilharziella; Ornithobilharzia and Austrobilharzia (called 'Sinobilharzia' in the tree based on morphology). However, as a result of topological differences, the interpretation of the evolution of the family, including the adoption of intermediate and definitive hosts, also changes. Of particular interest is whether the move from avian to mammalian definitive hosts was a single event. For this to be resolved, the true identity of the sister group to Schistosoma must be identified.

While Schistosoma has long been a subject of study, a clear phylogeny for the genus has remained elusive. There are discrepancies in our understanding of the radiation of Schistosoma, but this stems largely from later efforts building on earlier, relatively poorly sampled attempts, in a fragmented manner. There has rarely been full complementarity between the various studies undertaken, such that some genes are sampled for some taxa but not for all. Johnston et al. (1993) and Littlewood \& Johnston (1995) used almost complete ssrDNA and partial lsrDNA respectively to estimate the interrelationships of exemplar taxa from the main Schistosoma species groups. Barker \& Blair (1996) incorporated more species, but used shorter ssrDNA and lsrDNA fragments. Shorter, more variable regions of DNA from nuclear ribosomal internal transcribed spacer region 2 (ITS2) and mitochondrial cytochrome oxidase I (COI), were also used to confirm species groups (Bowles, Blair \& McManus, 1995), although again, only a limited number of 


\section{A morphology}

Morand \& Müller-Graf (2000) after Carmichael (1984)

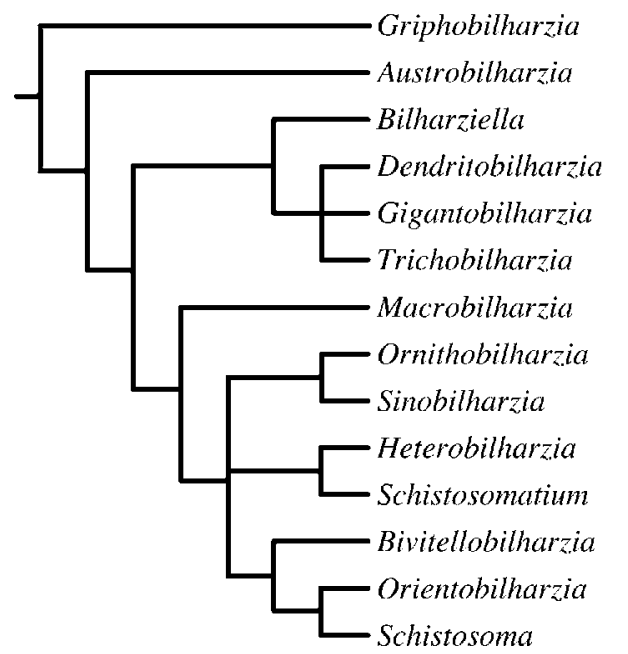

\section{B LSU rDNA}

Snyder \& I.oker (2000)

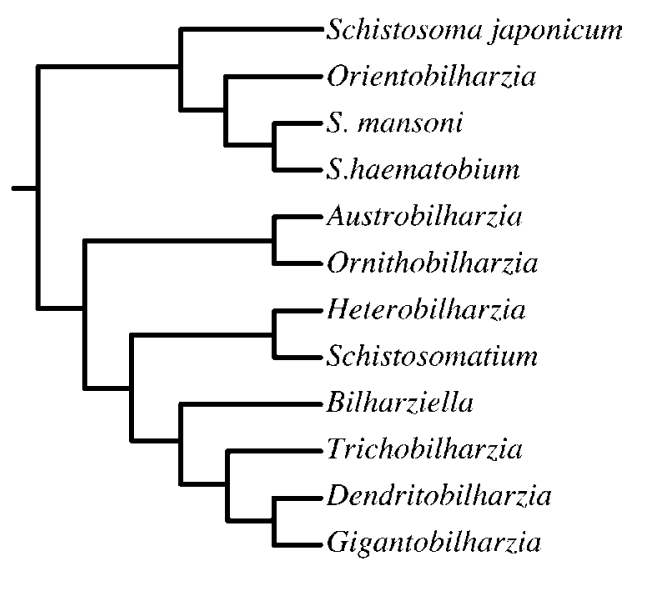

Fig. 1. Previously published phylogenies based on a cladistic analysis of morphology (recoding characters used by Carmichael, 1984; Morand \& Müller-Graf, 2000) and a molecular phylogenetic analysis of partial lsrDNA (Snyder \& Loker, 2000).

Note: Sinobilharzia refers to Austrobilharzia odhneri- One anomaly not explained by Morand \& Müller-Graf (2000) concerns this species. In Carmichael's analysis (Carmichael, 1984) Sinobilharzia represents a single species that was originally named Ornithobilharzia odhneri Faust, 1924, but subsequently reclassified as Sinobilharzia odhneri by Dutt \& Srivastava (1961). Farley (1971) then placed this species in Austrobilharzia, but Carmichael chose to analyse the species separately under the genus Sinobilharzia. In their analysis, Morand \& Müller-Graf (2000) used Carmichael's morphological matrix to produce a tree, but mapped specific morphological data from another species that they called Sinobilharzia crecci - we can find no reference for this species and suggest that they may have mistakenly used filinobilharzia crecci Liu \& Bai (1976) in their analysis. Although this has no effect on their analysis of Carmichael's matrix, it should be borne in mind that Sinobilharzia in the tree of Morand \& Müller-Graf (2000); shown in Fig. 1A refers to Austrobilharzia odhneri. Khalil (2002) synonymizes Sinobilharzia as Austrobilharzia. Indeed, Sinobilharzia, which has also been used for Schistosoma japonicum by Le Roux (1958), is no longer recognized.

exemplar taxa were included. Most recent studies have added gene fragments or additional taxa to particular clades within Schistosoma to test the position of individual taxa (e.g. Agatsuma et al. 2001, 2002; Blair et al. 1997), or to examine some of the biogeographic hypotheses suggested by Snyder \& Loker's (2000) scheme, e.g. Zhang et al. (2001) and Attwood et al. (2002 b). Few molecular studies have focused on the non-Schistosoma groups, although molecular methods to differentiate Trichobilharzia species are being developed (Dvorak et al. 2002).

Snyder \& Loker (2000) found Orientobilharzia among the Schistosoma lineages, suggesting that Schistosoma is paraphyletic. Blair, Davis \& Wu (2001) using the same data with the addition of more Schistosoma species, showed a single mammalian/avian split but without strong nodal support. A recent summary (Morgan et al. 2001) indicated that the Schistosoma phylogeny is not resolved leaving many questions unanswered, such as the position of $S$. incognitum. Also, while it now seems likely that the East Asian species are the earliest derived species in the Schistosoma clade, the position of Orientobilharzia, either within the East Asian clade or basal to the African and Indian species, remains problematic (Attwood et al. $2002 a$; Snyder \& Loker, 2000; Zhang et al. 2001).
Additional evidence for the phylogeny within Schistosoma has been gleaned from investigating complete mitochondrial genomes, and a remarkable split in the genus has been revealed (Le et al. 2000). The gene order of $S$. mansoni is quite different to that found in S. japonicum and S. mekongi and these 2 Asian schistosomes share the same gene order as that found in other trematodes and in cestodes, suggesting that they possess the plesiomorphic pattern. $S$. mansoni not only has a translocation of atp 6 and nad 2 when compared to $S$. japonicum, but also the gene order for nad 3 and nad 1 is reversed, and thus these data confirm the basal status of the East Asian Schistosoma. The position of the East Asian species is significant in distinguishing between different theories for the origin and subsequent radiation and dispersal of the schistosomes.

\section{Biogeography}

Two theories of Schistosoma origin have been proposed. A Gondwanan-origin (vicariance) hypothesis, based on snail host phylogeny and palaeontology, suggests members of the genus originated in Gondwanaland, with an ancestor rafting on the Indian plate to Asia 70-150 MYA and that Schistosoma 
transferred to South America 80-120 MYA before continental drift split Gondwanaland (Davis, 1980). The molecular evidence so far refutes this scenario. Firstly, Després et al. (1993) using restriction fragment length polymorphisms (RFLPs) of mitochondrial DNA fragments found that the genetic differentiation between African and American populations of $S$. mansoni was no greater than within African populations, suggesting a recent transfer of the parasite to S. America, associated with the slave trade. Secondly, Snyder \& Loker (2000) found S. japonicum and Orientobilharzia at the base of the Schistosoma phylogeny and proposed an alternative, Asian, hypothesis. They proposed (and subsequently suggested dates by referring to the historical record of the vertebrate hosts (Morgan et al. 2001)), that an ancestral schistosome dispersed to Africa 12-19 MYA via widespread mammal migration from Asia. The Schistosoma ancestor remaining in Asia radiated as the $S$. japonicum species group. In Africa the lineage diverged into the $S$. mansoni and S. haematobium groups and an $S$. indicum ancestor migrated back to India, possibly with early humans and their animals. S. mansoni dispersed to South America about 500 YA via the transport of African slaves (Després et al. 1993). Dating such events is highly problematic. Barker \& Blair (1996) rejected the use of a molecular clock based on partial lsrDNA, and Snyder \& Loker (2000) recognized the whole exercise as highly speculative. Other dates have been proposed for these various splits, but all depend on the acceptance of molecular clocks (Després et al. 1992; Morgan et al. 2001) that are at best highly erratic and that have been employed without estimating confidence intervals (e.g. Cutler, 2000; Rambaut, 2000). Attwood (2001), Attwood \& Johnston (2001) and Attwood et al. (2002b) also discussed biogeographical predictions for Schistosoma which are concordant with an Asian origin, based on intermediate host phylogeography and the late Caenozoic evolution of the main rivers in Asia. Attwood (2002 b) used partial 18S, 28S and mitochondrial 16S rRNA gene sequences to estimate a phylogeny for the East Asian species which was independent of a molecular clock hypothesis but does rely on, as yet, incomplete palaeogeographical data.

\section{This study}

A robust and comprehensive phylogeny is required to enable us to stabilize the taxonomy, to identify taxonomically useful characters, to investigate the biogeography and the origin of the schistosomes, and to reveal other unique features of this important group, including host-specificity, host-switching, and the evolution of sexual dimorphism. Many of these questions have recently been subjects of investigation and there have been attempts to construct 'supertrees' based on various previous phylogenetic estimates from smaller trees with fewer taxa (Morand
\& Müller-Graf, 2000). However, an estimate of the phylogeny based on a fully complementary multigene dataset is required. This paper extends on previous studies by using 2 nuclear and 1 mitochondrial gene. Although previous work has resolved some schistosomatid relationships with 1srDNA and ssrDNA sequences treated individually, it is clear that a combination of these data works well among platyhelminth groups in general, and particularly amongst neodermatan flatworms (e.g. Olson et al. 2001; Olson \& Littlewood, 2002; Olson et al. manuscript submitted). However, rather than relying on partial lsrDNA alone, there is growing evidence that combining the complete sequences of both genes adds stability and resolution at a number of levels within and between metazoan taxa (Mallatt \& Winchell, 2002; Medina et al. 2001), including platyhelminths (Lockyer, Olson \& Littlewood, 2003). Additionally, almost complete mitochondrial COI was sequenced in order to provide greater resolution among more closely related taxa. These 3 genes were sequenced from 30 taxa, including at least 1 representative from 10 of the 13 known genera of the Schistosomatidae and 17 of the 20 recognized Schistosoma species.

\section{MATERIALS AND METHODS}

\section{Taxa sampled and choice of outgroup}

Twenty-nine schistosomatid species and one sanguinicolid for outgroup rooting were sampled. Previous morphological and molecular phylogenetic estimates of digenean interrelationships have indicated strongly that the Sanguinicolidae are the sister group to the Schistosomatidae within the superfamily Schistosomatoidea (see Cribb et al. 2001). Recent work (D.T.J.L. \& P.D.O., unpublished results) has indicated that sanguinicolids are quite divergent from the schistosomatids, exhibiting relatively long branches for both ssrDNA and lsrDNA. Nevertheless, each selected gene partition was sequenced from the basal sanguinicolid Chimaerohemecus trondheimensis, its position based on analyses of digenean interrelationships using full ssrDNA and partial lsrDNA (D. T.J.L., P.D.O., unpublished results). An, as yet undescribed, sanguinicolid, used elsewhere for ssrDNA and lsrDNA analyses of platyhelminth relationships (Lockyer, Olson \& Littlewood, 2003), added additional information for outgroup rooting. Although the COI fragment could not be amplified from this second outgroup taxon, all ingroup topologies of ssrDNA and lsrDNA trees were identical with one or two outgroups, so the analyses were restricted to rooting against $C$.trondheimensis alone. If suggestions that the Spirorchidae are in fact the sister group to the Schistosomatidae (Platt \& Brooks, 1997) can be confirmed, additional molecular sampling from this family may be worthwhile. No spirorchids were available for the present analysis. 
All major schistosomatid genera were sampled except Macrobilharzia, Bivitellobilharzia, Filinobilharzia and Griphobilharzia. These taxa are parasites of protected or rare vertebrate hosts and one, Griphobilharzia, has remained elusive since its original description from the freshwater crocodile (Platt et al. 1991). Among the genus Schistosoma, every species was sampled except $S$. hippopotami and $S$. edwardiense, both parasites of Hippopotamus amphibious L., another protected species. Unfortunately, there were insufficient female (most readily identifiable) specimens of S. ovuncatum (Attwood et al. 2002a) available for the present study. The full classification of the Schistosomatidae according to the latest keys (Khalil, 2002) is replicated in Table 1. The same table gives full details of the taxa sampled here, including authorities and sources.

\section{DNA extraction and gene sequencing}

Total genomic DNA was extracted from liquid nitrogen-frozen or ethanol-preserved specimens using standard proteinase $\mathrm{K}$, phenol-chloroform extraction techniques (Sambrook, Fritsch \& Maniatis, 1989) or DNeasy $^{\text {TM }}$ Tissue kit (Qiagen) according to the manufacturer's protocol. The $25 \mu \mathrm{l}$ amplifications were performed with $3-5 \mu 1$ of genomic extract ( $\sim 10 \mathrm{ng}$ ) using Ready-To-Go PCR beads (Amersham Pharmacia Biotech) each containing 1.5 U Taq Polymerase, $10 \mathrm{~mm}$ Tris- $\mathrm{HCl}(\mathrm{pH} 9 \cdot 0), 50 \mathrm{~mm} \mathrm{KCl}$, $1.5 \mathrm{mM} \mathrm{MgCl}_{2}, 200 \mu \mathrm{M}$ each dNTP and stabilisers including BSA; and $0.4 \mu \mathrm{M}$ of each PCR primer. The complete lsrDNA was amplified in 3 overlapping sections using the primer combinations U178+ $\mathrm{L} 1642$, U1148 + L2450 and U1846+L3449 (see Table 2). PCR conditions used were: $2 \mathrm{~min}$ denaturation at $94{ }^{\circ} \mathrm{C}$; 40 cycles of $30 \mathrm{sec}$ at $94{ }^{\circ} \mathrm{C}$, $30 \mathrm{sec}$ at $52{ }^{\circ} \mathrm{C}$ and $2 \mathrm{~min}$ at $72{ }^{\circ} \mathrm{C}$; followed by a final 7 min extension at $72^{\circ} \mathrm{C}$. Where necessary to obtain a product, the stringency was reduced by adding $\mathrm{MgCl}_{2}$ to a final concentration of $2.5 \mathrm{~mm}$ or by reducing the annealing temperature to $50{ }^{\circ} \mathrm{C}$. Amplification of mitochondrial cytochrome oxidase subunit 1 (CO1) was performed using the primers Cox1_Schist_5' and Cox1_Schist_3' (see Table 2) with the same PCR conditions as above. Complete sequencing of ssrDNA was performed as described previously (Littlewood et al. 1999).

PCR products were purified with Qiagen Qiaquick columns, cycle-sequenced directly using ABI BigDye chemistry, ethanol-precipitated and run on an ABI prism 377 automated sequencer. A variety of internal primers were used to obtain the full sequence of each fragment from both strands (see Table 2). Sequences were assembled and edited using Sequencher ver 3.1.1 (GeneCodes Corp.) and submitted to EMBL/GenBank (see Table 1 for accession numbers). In all cases complete lsrDNA, ssrDNA and CO1 were sequenced, except for conserved regions at both $5^{\prime}$ and $3^{\prime}$ ends that were targeted for primer design.

\section{Sequence alignment and phylogenetic analyses}

ssrDNA and lsrDNA sequences were each aligned initially with the aid of ClustalX using default parameters (Jeanmougin et al. 1998), and alignments then refined by eye with MacClade ver. 4.03 (Maddison \& Maddison, 2000). CO1 sequences were aligned with reference to the open-reading frame and the inferred amino acid sequences. Individual gene alignments were concatenated in MacClade, ambiguously aligned positions excluded and data partitions defined.

Maximum parsimony (MP) and maximum likelihood (ML) analyses were performed using PAUP* ver. 4.0b10 (Swofford, 2002) and the resulting networks rooted with the outgroup taxon. Each gene was analysed both independently and combined using MP, ML and also Bayesian inference (BI) using the program MrBayes (Huelsenbeck, 2000). Mitochondrial COI sequences were analysed only as nucleotides but were investigated to see whether trees differed in topology when using only first and second codon positions or all 3 positions, in order to best reflect the signal at nonsynonymous sites. Analyses by MP were performed using a heuristic search strategy (1000 search replicates), random-addition of sequences and tree-bisection-reconnection (TBR) branch-swapping options. All characters were run unordered and equally weighted. Gaps were treated as missing data. Nodal support was assessed by bootstrap resampling in MP (1000 replicates) and ML (100 replicates). Nodal support from majority-rule consensus trees found with BI were also utilized. In order to test whether there was significant conflict between the data partitions prior to combining them the criteria of conditional combination of independent data sets (Huelsenbeck, Bull \& Cunningham, 1996; Cunningham, 1997) were examined using the incongruence length-difference (Farris et al. 1995) test as implemented in PAUP*. The test was performed with maximum parsimony, 10 heuristic searches (random sequence addition, TBR branchswapping) each for 100 homogeneity-replicates on informative sites only (Lee, 2001).

A suitable nucleotide substitution model was estimated using Modeltest (Posada \& Crandall, 1998), which showed a general time reversible $(G T R)$ model including estimates of invariant sites $(I)$ and amongsite rate heterogeneity $(G)$ for each individual and combined data set. In calculating maximum likelihood trees, values of $I$ and $G$ were set to those estimated by Modeltest but substitution rate parameters were free to vary and nucleotide frequencies used were empirical.

Bayesian inference (BI) of phylogeny was estimated using the following nucleotide substitution 
Table 1. List of taxa and sequences used in this study and their geographical origin

(Avian (A) or mammalian (M) vertebrate host indicated. See Fig. 5 for list of mollusc hosts. Previously unreported sequences are marked §.)

\begin{tabular}{|c|c|c|c|c|c|c|}
\hline \multirow[b]{2}{*}{ Classification } & \multirow[b]{2}{*}{ Source } & \multicolumn{2}{|c|}{ Vertebrate host } & \multicolumn{3}{|c|}{ GenBANK Accession No. } \\
\hline & & A & M & COI & ssrDNA & lsrDNA \\
\hline \multicolumn{7}{|l|}{ Schistosomatidae Stiles \& Hassall, 1898} \\
\hline \multirow{2}{*}{\multicolumn{7}{|c|}{$\begin{array}{l}\text { Schistosomatinae Stiles \& Hassall, } 1898 \\
\text { Austrobilharzia Johnston, } 1917\end{array}$}} \\
\hline & & & & & & \\
\hline Austrobilharzia terrigalensis Johnson, 1916 & $\begin{array}{l}\text { ex Batillaria australis; Rodd Point, Iron Cove, } \\
\text { Sydney Harbour, NSW, Australia. }\end{array}$ & $\checkmark$ & & AY157195§ & AY157223§ & AY157249§ \\
\hline Austrobilharzia variglandis (Miller \& Northup, 1926) & ex Larus delawarensis; Delaware, USA. & $\checkmark$ & & AY157196§ & AY157224§ & AY157250§ \\
\hline $\begin{array}{l}\text { Bivitellobilharzia Vogel \& Minning, } 1940 \\
\text { Heterobilharzia Price, } 1929\end{array}$ & No species sampled & & $\checkmark$ & & & \\
\hline Heterobilharzia americana Price, 1929 & $\begin{array}{l}\text { ex Mesocricetus auratus; (experimental infection) } \\
\text { NHM-409 original isolate from Louisiana, USA. }\end{array}$ & & $\checkmark$ & AY157192§ & AY157220§ & AY157246§ \\
\hline Macrobilharzia Travassos, 1922 & No species sampled. & $\checkmark$ & & & & \\
\hline \multicolumn{7}{|l|}{ Orientobilharzia Dutt \& Srivastava, 1955} \\
\hline Orientobilharzia turkestanicum (Skrjabin, 1913) & ex Ovis aries; Iran. & & $\checkmark$ & AY157200§ & AF442499 & AY157254§ \\
\hline \multicolumn{7}{|l|}{ Ornithobilharsia Odhner, 1912} \\
\hline Ornithobilharzia canaliculata (Rudolphi, 1819) & ex Larus delawarensis; Donley County, Texas, USA. & $\checkmark$ & & AY157194§ & AY157222§ & AY157248§ \\
\hline \multicolumn{7}{|l|}{ Schistosomatium Tanabe, 1923} \\
\hline Schistosomatium douthitti (Cort, 1915) & $\begin{array}{l}\text { ex Mesocricetus auratus; (experimental infection) } \\
\text { Indiana, USA. }\end{array}$ & & $\checkmark$ & AY157193§ & AY157221§ & AY157247§ \\
\hline \multicolumn{7}{|l|}{ Schistosoma Weinland, 1858} \\
\hline Schistosoma bovis (Sonsoni, 1876) & $\begin{array}{l}\text { ex Mus musculus; (experimental infection) original } \\
\text { isolate from Iranga, Tanzania. }\end{array}$ & & $\checkmark$ & AY157212§ & AY157238§ & AY157266§ \\
\hline Schistosoma curassoni Brumpt, 1931 & $\begin{array}{l}\text { ex Mesocricetus auratus; (experimental infection) } \\
\text { original isolate from Dakar, Senegal. }\end{array}$ & & $\checkmark$ & AY157210§ & AY157236§ & AY157264§ \\
\hline Schistosoma haematobium (Bilharz, 1852) & $\begin{array}{l}\text { ex Mesocricetus auratus; (laboratory infection) } \\
\text { NHM-3390, Village 10, Nigel delta, Mali. }\end{array}$ & & $\checkmark$ & AY157209§ & Z11976 & AY157263§ \\
\hline Schistosoma incognitum Chandler, 1926 & ex Bandicota indica; Phitsanulok, Thailand. & & $\checkmark$ & AY157201§ & AY157229§ & AY157255§ \\
\hline Schistosoma indicum Montgomery, 1906 & ex Bos taurus; Mymensingh, Bangladesh. & & $\checkmark$ & AY $157204 \S$ & AY157231§ & AY157258§ \\
\hline Schistosoma intercalatum Fisher, 1934 & $\begin{array}{l}\text { ex Mus musculus; (laboratory infection) } \\
\text { NHM-3188, San Antonio, São Tomé. }\end{array}$ & & $\checkmark$ & AY157208§ & AY157235§ & AY157262§ \\
\hline Schistosoma japonicum Katsurada, 1904 & $\begin{array}{l}\text { ex Mus musculus; (experimental infection) } \\
\text { isolate S15/90-19. Original isolate from the Philippines. }\end{array}$ & & $\checkmark$ & AF215860 & AY157226§ & AY157607§ \\
\hline Schistosoma leiperi Le Roux, 1955 & $\begin{array}{l}\text { ex Mesocricetus auratus; (experimental infection) } \\
\text { original isolate from South Africa. }\end{array}$ & & $\checkmark$ & AY157207§ & AY157234§ & AY157261§ \\
\hline Schistosoma malayensis Greer et al. 1988 & $\begin{array}{l}\text { ex Mus musculus; (experimental infection) } \\
\text { original isolate from Baling, Kedah, Malaysia }\end{array}$ & & $\checkmark$ & AY157198§ & AY157227§ & AY157252§ \\
\hline Schistosoma mansoni Sambon, 1907 & $\begin{array}{l}\text { ex Mus musculus; (experimental infection) } \\
\text { isolate NHM-3454/5/6. }\end{array}$ & & $\checkmark$ & AF216698 & M62652 & AY157173§ \\
\hline Schistosoma margrebowiei Le Roux, 1933 & $\begin{array}{l}\text { ex Mus musculus; (experimental infection) } \\
\text { lab strain isolate NHM-3295. Original }\end{array}$ & & $\checkmark$ & AY157206§ & AY157233§ & AY157260§ \\
\hline
\end{tabular}


Schistosoma mekongi Voge, Buckner \& Bruce, 1978

Schistosoma nasale Rao, 1933

Schistosoma rodhaini Brumpt, 1931

Schistosoma sinensium Bao, 1958

Schistosoma spindale Montgomery, 1906

Griphobilharziinae Platt, Blair, Purdie \& Melville, 1991

Griphobilharzia Platt, Blair, Purdie \& Melville, 1991 Bilharziellinae Price, 1929

Bilharziella, Looss, 1899

Bilharziella polonica (Kowalewski, 1895)

Filinobilharzia Liu \& Bai, 1976

Trichobilharzia, Skrjabin \& Zakharow, 1920

Trichobilharzia ocellata (La Valette, 1855)

Trichobilharzia regenti Horak, Kolarova \&

Dvorak, 1998

Trichobilharzia szidati Neuhaus, 1952

Gigantobilharziinae Mehra, 1940

Dendritobilharzia Skrjabin \& Zakharow, 1920

Dendritobilharzia pulverulenta (Braun, 1901)

Gigantobilharzia Odhner, 1910

Gigantobilharzia huronensis Najim, 1950

Sanguinicolidae

Chimaerohemecus trondheimensis van der Land, 1967 ex Mus musculus; (experimental infection)

original isolate from Denwood Farm

$\mathrm{Nr}$ Lusaka, Zambia.

ex Mus musculus; (experimental infection)

originally isolated from Neotricula

aperta, Khong Island, Laos.

ex Capra hircus; Sri Lanka.

ex Mus musculus; (experimental infection)

lab strain (NHM).

ex Mus musculus; (experimental infection)

originally isolated from Tricula sp.

Mianzhu, Sichuan, China.

ex Mus musculus; (experimental infection) NMH 1630

original isolate from Indoplanorbis

exustus from Sri Lanka.

No species sampled.

ex Anas platyrhynchus; Kherson Oblast, Ukraine.

No species sampled.

ex Lymnaea stagnalis; Germany.

ex Radix peregra; (experimental infection);

Horak Lab., Prague, Czech Rep.

ex Lymnaea stagnalis; (experimental infection);

Horak Lab., Prague, Czech Rep.

ex Gallus gallus; (experimental infection),

Bernallio County, New Mexico, USA.

ex Agelaius phoeniceus; Winnebago County, Wisconsin, USA.

ex Chimaera monstrosa; Korsfiorden, near Bergen, Norway.

\begin{tabular}{|c|c|c|c|}
\hline$\checkmark$ & AY157211§ & AY157237§ & AY157265 \\
\hline$\checkmark$ & AY157199§ & AY157228§ & AY157253§ \\
\hline$\checkmark$ & AY157205§ & AY157232§ & AY157259§ \\
\hline$\checkmark$ & AY157202§ & AY157230§ & AY $157256 \S$ \\
\hline$\checkmark$ & AY157197§ & AY157225§ & AY157251§ \\
\hline$\checkmark$ & AY157203§ & Z11979 & AY157257§ \\
\hline
\end{tabular}

AY157186§ AY157214§ AY157240§

AY157189§ AY157217\$ AY157243\$ AY157190§ AY157218§ AY157244§

AY157191§ AY157219§ AY157245§

AY157187§ AY157215§ AY157241§

AY157188§ AY157216§ AY157242§

AY157185§ AY157213§ AY157239§ 
Table 2. Primers used for PCR amplification and sequencing of complete lsrDNA and CO1

(See Littlewood et al. (1999) for ssrDNA amplification and sequencing primers.)

\begin{tabular}{|c|c|}
\hline $\begin{array}{l}\text { Amplification } \\
\text { and sequencing }\end{array}$ & Primer sequence $\left(5^{\prime}-3^{\prime}\right)$ \\
\hline \multicolumn{2}{|l|}{ lsrDNA } \\
\hline U178 & GCACCCGCTGAAYTTAAG \\
\hline L1642 & CCAGCGCCATCCATTTTCA \\
\hline $\mathrm{U} 1148$ & GACCCGAAAGATGGTGAA \\
\hline $\mathrm{L} 2450$ & GCTTTGTTTTAATTAGACAGTCGGA \\
\hline U1846 & AGGCCGAAGTGGAGAAGG \\
\hline L3449 & ATTCTGACTTAGAGGCGTTCA \\
\hline \multicolumn{2}{|l|}{ COI } \\
\hline Cox1_schist_5' & TCTTTRGATCATAAGCG \\
\hline Cox1_schist_3' & TAATGCATMGGAAAAAAACA \\
\hline \multicolumn{2}{|c|}{ Additional sequencing primers } \\
\hline \multicolumn{2}{|l|}{ lsrDNA } \\
\hline $300 \mathrm{~F}$ & CAAGTACCGTGAGGGAAAGTTG \\
\hline $300 \mathrm{R}$ & CAACTTTCССТCACGGTACTTG \\
\hline EDC2 & CCTTGGTCCGTGTTTCAAGACGGG \\
\hline $900 \mathrm{~F}$ & CCGTCTTGAAACACGGACCAAG \\
\hline $1200 \mathrm{~F}$ & CCCGAAAGATGGTGAACTATGC \\
\hline $1200 \mathrm{R}$ & GCATAGTTCACCATCTTTCGG \\
\hline $1600 \mathrm{~F}$ & AGCAGGACGGTGGCCATGGAAG \\
\hline U2229 & TACCCATATCCGCAGCAGGTCT \\
\hline $\mathrm{L} 2230$ & AGACCTGCTGCGGATATGGGT \\
\hline $\mathrm{U} 2562$ & AAACGGCGGGAGTAACTATGA \\
\hline L2630 & GGGAATCTCGTTAATCCATTCA \\
\hline $\mathrm{U} 2771$ & AGAGGTGTAGGATARGTGGGA \\
\hline L2984 & CTGAGCTCGCCTTAGGACACCT \\
\hline $\mathrm{U} 3119$ & TTAAGCAAGAGGTGTCAGAAAAGT \\
\hline U3139 & AAGTTACCACAGGGATAACTGGCT \\
\hline LSU3_4160 & GGTCTAAACCCAGCTCACGTTCCC \\
\hline L3358 & AACCTGCGGTTCCTCTCGTACT \\
\hline \multicolumn{2}{|l|}{$\mathrm{COI}$} \\
\hline $\mathrm{CO} 1560 \mathrm{Fa}$ & TTTGATCGTAAATTTGGTAC \\
\hline $\mathrm{CO} 1560 \mathrm{Fb}$ & TTTGATCGGAATTTTGGTAC \\
\hline CO1560R & GCAGTACCAAATTTACGATC \\
\hline $\mathrm{CO} 1800 \mathrm{~F}$ & CATCATATGTTTATGGTTGG \\
\hline $\mathrm{CO} 1800 \mathrm{Ra}$ & CCAACCATAAACATATGATG \\
\hline $\mathrm{CO} 1800 \mathrm{Rb}$ & CCAACCATAAACATGTGATG \\
\hline
\end{tabular}

parameters: lset $n s t=6$, rates $=$ invgamma, ncat $=4$, shape $=$ estimate,$\quad$ inferrates $=$ yes and basefreq $=$ empirical, that approximates to a $G T R+I+G$ model as above. Posterior probabilities were approximated over 200000 generations, log-likelihood scores plotted and only the final $85 \%$ of trees where the loglikelihood had reached a plateau were used to produce the consensus tree.

In order to include more sites and test further the interrelationships of the Schistosoma species, a subset of the entire dataset comprising only the Schistosoma (but including Orientobilharzia) was analysed rooting against the most basal, East Asian clade, as determined in the full analyses.

Final tree topologies were tested against previous hypotheses of interrelationships by using ML alone on the combined data set to find the best constrained trees, and then applying the Shimodaira-Hasegawa test (Shimodaira \& Hasegawa, 1999) as implemented in PAUP* with full optimization and 1000 bootstrap replicates, testing within and between the constrained and unconstrained topologies.

\section{Character mapping and interpretation}

The morphological character matrix of Carmichael (1984) (based on personal observations of many schistosomatid species, as well as on an extensive review of literature including Farley (1971)) was adapted, in order to interpret our molecular estimate of phylogeny in the context of morphology. Carmichael's matrix of 24 characters was taken in its entirety, but taxa not sampled in this study, namely Old and New World Macrobilharzia and Bivitellobilharzia and 'Sinobilharzia', were omitted (see Fig. 1 legend). Characters that changed unambiguously were mapped on our phylogeny using MacClade 
Table 3. Maximum likelihood parameter estimates

(All estimates are based on a general time reversible model of nucleotide substitution incorporating estimates of among-site rate variation (ASRV), estimated proportion of invariant sites ( $\mathrm{Inv}-\mathrm{E})$, transition rates (Ts), transversion rates ( $\mathrm{Tv}$ ) and alpha shape parameter estimate of the gamma distribution $(\alpha)$. $\mathrm{CO}_{12}$ and $\mathrm{CO}_{123}$ indicate analyses using only the first two codon positions for cytochrome oxidase 1 , and that using all 3 positions, respectively.)

\begin{tabular}{|c|c|c|c|c|c|c|c|c|}
\hline \multirow[b]{2}{*}{ Data partition } & \multicolumn{2}{|l|}{ ASRV } & \multicolumn{2}{|l|}{ Ts } & \multicolumn{4}{|l|}{$\mathrm{Tv}$} \\
\hline & $\alpha$ & Inv-E & $\mathrm{AG}$ & $\mathrm{CT}$ & $\mathrm{AC}$ & AT & GC & GT \\
\hline \multicolumn{9}{|l|}{ All taxa } \\
\hline ssrDNA & $0 \cdot 652$ & $0 \cdot 539$ & $5 \cdot 021$ & $7 \cdot 039$ & $0 \cdot 901$ & $1 \cdot 842$ & $0 \cdot 660$ & $1 \cdot 000$ \\
\hline lsrDNA & 0.639 & $0 \cdot 494$ & $4 \cdot 260$ & $5 \cdot 755$ & $0 \cdot 508$ & $1 \cdot 955$ & $0 \cdot 342$ & $1 \cdot 000$ \\
\hline $\mathrm{CO}_{12}$ & $0 \cdot 421$ & $0 \cdot 453$ & $8 \cdot 798$ & $8 \cdot 779$ & $0 \cdot 639$ & $1 \cdot 980$ & $2 \cdot 441$ & $1 \cdot 000$ \\
\hline $\mathrm{CO}_{123}$ & $0 \cdot 449$ & $0 \cdot 370$ & $18 \cdot 815$ & $25 \cdot 656$ & $1 \cdot 723$ & $2 \cdot 421$ & $8 \cdot 636$ & $1 \cdot 000$ \\
\hline ssrDNA + lsrDNA + $\mathrm{CO}_{123}$ & $0 \cdot 351$ & $0 \cdot 589$ & $5 \cdot 929$ & $2 \cdot 879$ & $0 \cdot 276$ & $2 \cdot 401$ & $0 \cdot 339$ & $1 \cdot 000$ \\
\hline \multicolumn{9}{|l|}{ Schistosoma only } \\
\hline ssrDNA + lsrDNA $+\mathrm{CO}_{123}$ & $0 \cdot 438$ & $0 \cdot 743$ & $7 \cdot 101$ & $2 \cdot 648$ & $0 \cdot 133$ & $2 \cdot 762$ & $0 \cdot 263$ & $1 \cdot 000$ \\
\hline
\end{tabular}

(Maddison \& Maddison, 2000) and treated as unweighted and unordered but were not recoded.

To further interpret the phylogeny, the HostParasite Database (H-PD) of The Natural History Museum (Gibson \& Bray, 1994), see www.nhm.ac.uk/zoology/hp-dat.htm, was used to code the snail genera used as intermediate hosts by the taxa studied here as well as those snail genera used by the other schistosomatid species not available in our molecular study. Using a variety of literature, including Carmichael (1984), Farley (1971) and the H-PD, the biogeographical distribution of species and genera included in our phylogenetic estimates was also examined. It should be noted that relying on the literature may incorporate certain errors, particularly where authors have misidentified parasite or host taxa. The best test for host associations is full, experimentally determined, life-cycle information but this is unavailable for most taxa. Finally, mitochondrial gene arrangements, based on published and unpublished observations of taxa used in this study were coded or inferred according to phylogenetic position and mapped on the phylogeny.

\section{RESULTS}

Accession numbers for each gene sequenced are shown in Table 1. Only ML solutions are presented for each gene, as BI methods yielded identical tree topologies throughout and MP produced only minor deviations in some cases. The full $G T R+I+G$ model parameters for each data partition are shown in Table 3. Major associations for each individual gene are presented below, but the full detail of species interrelationships is restricted to the combined evidence solution (Fig. 5 below).

\section{Cytochrome oxidase I}

A total of 1139 sites were available for alignment, of which 1122 were unambiguously aligned. Of the aligned positions 524 were constant and 498 parsimony informative. Removing third codon positions resulted in 748 included positions, of which 496 were constant and 180 parsimony informative. Phylogenetic estimates using the first 2 and all 3 codon positions are shown in Fig. 2A and B, respectively. The trees are almost identical in topology, suggesting none or insignificant levels of bases saturation, but with longer branch lengths for all taxa and greater resolution among the more derived Schistosoma taxa when all 3 positions were included (Fig. 2B). Dendritobilharzia falls as the most basal taxon with other bird schistosomes radiating first with a (Bilharziella+ Gigantobilharzia + Trichobilharzia) clade and then the (Ornithobilharzia + Austrobilharzia) clade. The mammalian schistosomes are split into 2 major clades, namely (Heterobilharzia + Schistosomatium) and (Schistosoma + Orientobilharzia).

Where multiple exemplars of genera were sampled, only Schistosoma appears non-monophyletic, due to the placement of Orientobilharzia. All schistosome species appear to be well differentiated from one another, in terms of molecular distance, except Trichobilharzia szidati and T. ocellata, which are almost identical. For COI these taxa differ in 9 bases out of $1125 \mathrm{bp}(0 \cdot 008)$ and all differences are at synonymous sites. Poorly resolved nodes, as judged by relatively low Bayesian support, include the relative placement of the bird schistosome genera, the (Ornithobilharzia + Austrobilharzia) clade, and the most derived members of the African Schistosoma. Otherwise the gene provides a high proportion of informative positions, at least as judged by parsimony, for its relatively short length.

\section{$\operatorname{ss} \mathrm{DNA}$}

A total of 1937 sites were available for alignment, of which 1831 were unambiguously aligned. Of the aligned positions 1526 were constant and 145 parsimony informative. The phylogenetic estimate 
A CO1 (12)

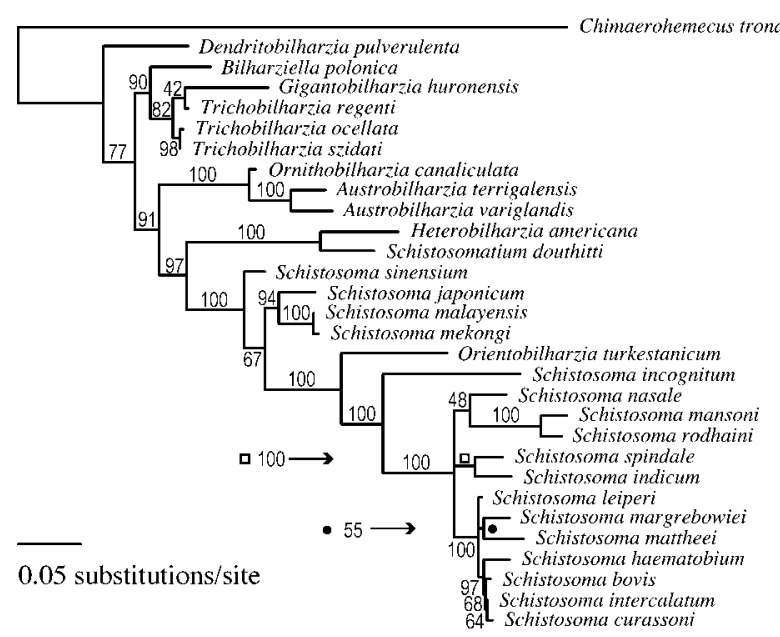

\section{SSU rDNA}

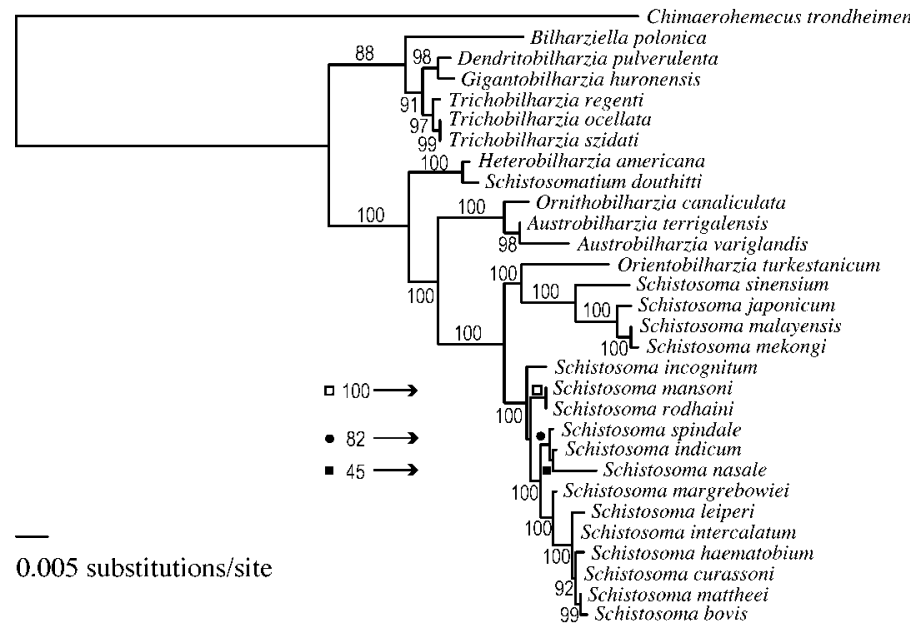

B CO1 (123)

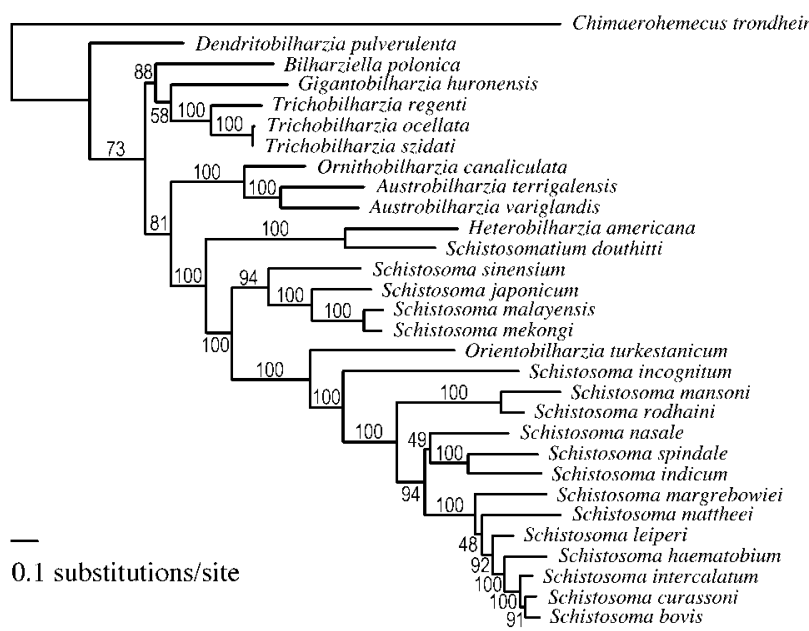

D LSU rDNA

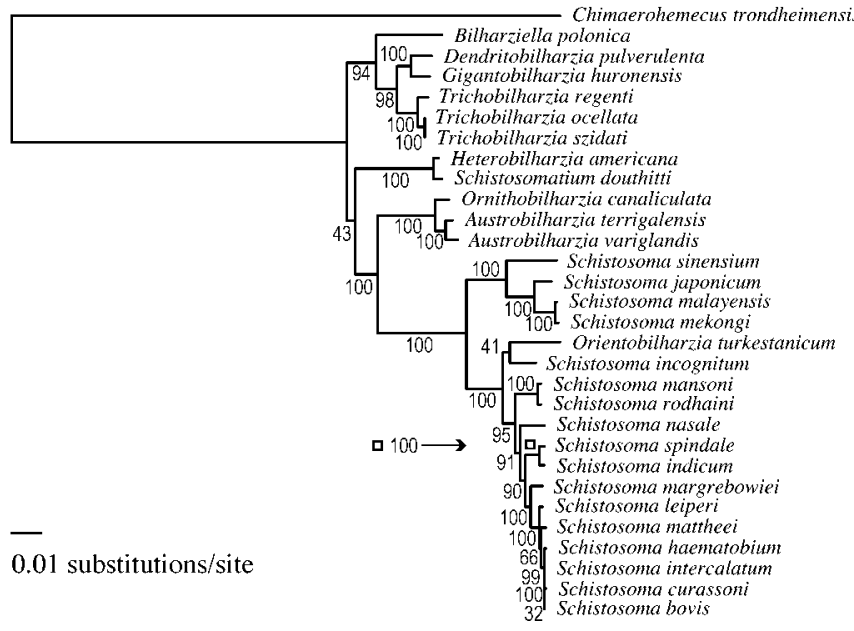

Fig. 2. Maximum likelihood estimates of the interrelationships of the Schistosomatidae from individual gene fragments. (A) Mitochondrial CO1 using only the first 2 codon positions. (B) Mitochondrial CO1 using all 3 codon positions. (C) Nuclear small subunit rDNA. (D) Nuclear large subunit rDNA. Nodal support values are posterior probabilities (expressed as percentages) from Bayesian analyses for each of the same data partitions. 
afforded by this gene is shown in Fig. 2C. In contrast to COI, Dendritobilharzia did not appear as the most basal taxon. Instead, a larger clade including (Dendritobilharzia + Bilharziella + Gigantobilharzia + Trichobilharzia) occupies this position. Also in contrast to COI, the next clade is (Heterobilharzia+ Schistosomatium) making the mammal schistosomes paraphyletic and giving (Ornithobilharzia+ Austrobilharzia) as the sister group to (Schistosoma + Orientobilharzia). It is noteworthy that the interrelationships within Schistosoma are almost identical to COI although Orientobilharzia falls in the same clade as the East Asian Schistosoma (S. sinensium, $S$. japonicum, S. malayensis and S. mekongi), rather than basal to the African and Indian species. In the case of Trichobilharzia, T. szidati and T. ocellata differ by just 1 base change in 1868 bp (0.0005).

\section{$\operatorname{ls} \mathrm{DNA}$}

A total of 3950 sites were available for alignment, of which 3765 were unambiguously aligned. Of the aligned positions 2900 were constant and 470 parsimony informative. The tree is shown in Fig. 2D and is almost identical in topology to that of ssrDNA except in one important aspect. As with COI, Orientobilharzia groups with $S$. incognitum, although with poor nodal support. Poor nodal support also characterizes the relative position of the (Heterobilharzia + Schistosomatium) clade and the interrelationships of the most derived African Schistosoma. In the case of Trichobilharzia, T. szidati and T. ocellata differ by 7 base changes in $3856 \mathrm{bp}(0 \cdot 0018)$.

\section{Combined COI, ssrDNA and lsrDNA}

The partition homogeneity test (ILD; incongruence length difference test), using 100 test replicates including parsimony informative sites only, indicated no significant difference in signal between the 3 genes for the ingroup $(P=0.09)$, and therefore passed a test for conditional combination of independent datasets. Considering that this test has been demonstrated to fail in detecting congruence when dealing with heterogeneous datasets, such as mitochondrial and nuclear gene sequences, the fact that no significant difference was found, adds greater confidence in combining our data (Dowton \& Austin, 2002). The combined data were analysed in full, and also for the (Schistosoma + Orientobilharzia) clade alone (ILD; $P=0 \cdot 65)$. Results of the full analysis are shown in Fig. 3. The avian clade is the same as with ssrDNA and lsrDNA alone, and the interrelationships of these schistosomes remains (Bilharziella (Trichobilharzia, (Dendritobilharzia, Gigantobilharzia))). The next 2 major clades appear as with ssrDNA and lsrDNA individually, but very poor nodal support means that the true position of (Heterobilharzia + Schistosomatium) may not be fully resolvable with these data alone. However, even with this node unresolved, it appears that mammalian schistosomes are paraphyletic and, as with the individual rRNA genes, the full analysis including COI resolves the bird schistosome clade (Ornithobilharzia + Austrobilharzia) as the sister group to the (Schistosoma+Orientobilharzia) clade. It is clear that Orientobilharzia turkestanicum is a member of the Schistosoma clade. The Schistosoma split into 2 lineages, the East Asian species and the rest. Within the East Asian clade, $S$. sinensium was the first to diverge, followed by $S$. japonicum. Orientobilharzia and S. incognitum separate the East Asian Schistosoma from the remaining schistosomes, but the relatively poor nodal support for $S$. incognitum suggests it may occupy a clade with Orientobilharzia (as suggested, also weakly, by the 1srDNA analysis). Of the remaining taxa, $S$. mansoni and $S$. rodhaini form a well-supported clade as do $S$. spindale and $S$. indicum. $S$. nasale is very weakly supported (by bootstrap analysis) as the sister group to $S$. spindale and S. indicum in the full analysis and its position remains unresolved with these and the clade of more derived taxa in the analysis of Schistosoma taxa alone. Indeed, little resolution was gained in analysing Schistosoma alone (results not shown). Only an additional 127 sites were re-included in the alignment (3 for CO1; 46 for ssrDNA; 78 for lsrDNA) and the topology within the (Schistosoma + Orientobilharzia) clade remained essentially the same as with the full analysis except that the relationships between the 3 most derived taxa were marginally better supported as $(S$. intercalatum $(S$. curassoni, $S$. bovis)) by both bootstrap analysis using maximum likelihood and the proportion of best Bayesian trees supporting the nodes.

\section{Constraint analyses}

Constraint analyses were performed in order to test whether the combined data set argued significantly against specific topologies that were different from the fully resolved, unconstrained solution provided by ML, MP and BI (shown in Fig. 3). In particular to test: (a) the avian schistosomes as a monophyletic clade; (b) the mammalian schistosomes as monophyletic; (c) the major avian clade including Bilharziella as the sister group to Schistosoma; (d) the (Heterobilharzia + Schistosomatium) clade as the sister group to Schistosoma (a slight variation on simply holding mammalian schistosomes as monophyletic); (e) Orientobilharzia and S. incognitum as monophyletic group, as suggested by lsrDNA alone (Fig. 2D); (f) Orientobilharzia belonging in a clade with the East Asian Schistosoma as suggested by ssrDNA alone (Fig. 2C); (g) the 'indicum' species group as monophyletic. Results are shown in Table 4. Of all of these permutations 2 hypotheses are clearly rejected by the full implementation of the Shimodaira-Hasegawa test. These were that Orientobilharzia falls in a clade 


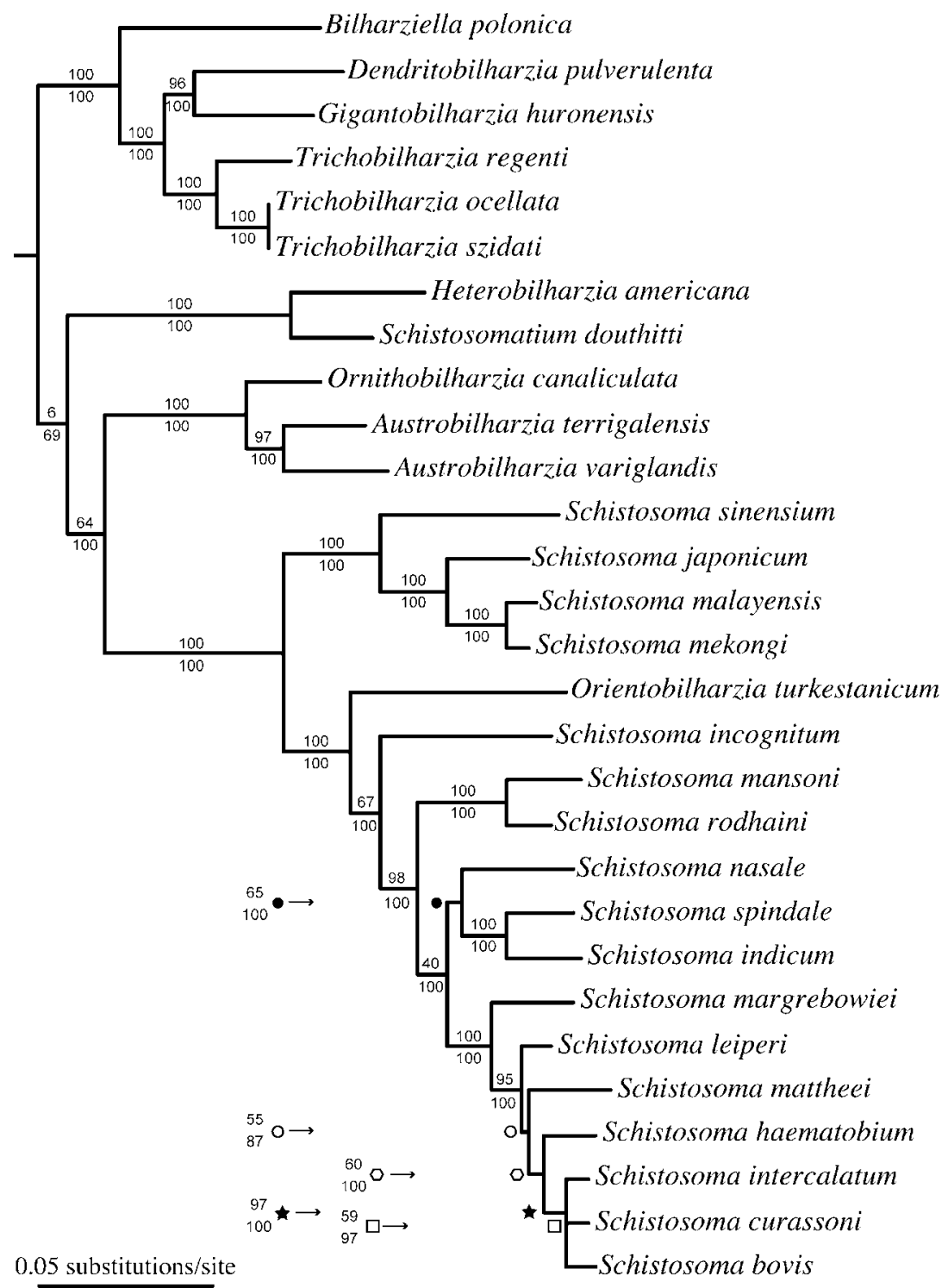

Fig. 3. Maximum likelihood estimate of the interrelationships of the Schistosomatidae from the combined analysis of data partitions (ssrDNA, lsrDNA and CO1 employing all 3 codon positions). Nodal support values are bootstrap values shown above $(n=100)$, and Bayesian (posterior) probabilities expressed as percentages, shown below branches.

with the East Asian Schistosoma and that the 'indicum' species group is monophyletic; S. incognitum does not cluster with the other 'indicum' group species, but lies basal to the schistosomes of Africa and the Indian subcontinent.

\section{Mapping morphological characters}

Six morphological characters from Khalil (2002) were taken as synapomorphies for the Schistosomatidae and a seventh character inferred from our tree, namely the presence of a gynaecophoric canal; Morand \& Müller-Graf (2000) also recognized this character as plesiomorphic for the Schistosomatidae. All other characters were taken directly from Carmichael (1984) and mapped onto the combined evidence topology shown in Fig. 3 (see Fig. 4; character numbers corresponding to the codes used by Morand \& Müller-Graf (2000) are also indicated). Carmichael's thesis deals only at the genus level and so the combined evidence tree is reduced to reflect this. At this level, all clades are supported by at least two synapomorphies except the union of (Ornithobilharzia + Austrobilharzia), which is strongly supported by all molecular data but no obvious morphological traits. Support for the sister group status between (Ornithobilharzia + Austrobilharzia) and (Schistosoma + Orientobilharzia) emerges from 2 synapomorphies; vitelline follicles paired along a common caecum and the female common caecum being long and straight. Four synapomorphies unite Schistosoma and Orientobilharzia; the absence of Laurer's Canal and a cirrus; weak coiling of the ovary and possession of a small, globular seminal vesicle. 
Table 4. Results of constraint analyses on the combined data set testing for the likelihood of accepting alternative tree topologies

(See text for further details. Log likelihood values, their differences with respect to the unconstrained solution and the significance of the constraints tested by the Shimodaira-Hasegawa test as implemented in PAUP* on ML trees are indicated; $P<0.05$ indicates a significantly different topology $(\dagger)$.)

\begin{tabular}{|c|c|c|c|}
\hline Constraint* & $-\ln \mathrm{L}$ & diff. $-\ln L$ & $P$ \\
\hline Unconstrained & $34170 \cdot 47$ & & \\
\hline Avian schistosomes monophyletic & $34180 \cdot 50$ & $10 \cdot 03$ & $0 \cdot 522$ \\
\hline Mammalian schistosomes monophyletic & $34179 \cdot 01$ & $8 \cdot 54$ & $0 \cdot 564$ \\
\hline Bilharziella clade $^{1}$ as sister to Schistosoma & $34180 \cdot 50$ & $10 \cdot 03$ & $0 \cdot 522$ \\
\hline Heterobilharzia clade $^{2}$ as sister to Schistosoma & $34179 \cdot 01$ & $8 \cdot 54$ & $0 \cdot 564$ \\
\hline Orientobilharzia $+S$. incognitum monophyletic & $34186 \cdot 67$ & $16 \cdot 20$ & $0 \cdot 356$ \\
\hline Orientobilharzia + E. Asian Schistosoma monophyletic ${ }^{3}$ & $34212 \cdot 00$ & $41 \cdot 53$ & $0 \cdot 035^{\dagger}$ \\
\hline 'indicum' species group monophyletic ${ }^{4}$ & $34228 \cdot 01$ & $57 \cdot 54$ & $0 \cdot 006^{\dagger}$ \\
\hline
\end{tabular}

* ${ }^{1}$ Bilharziella, Dendritobilharzia, Gigantobilharzia and Trichobilharzia; ${ }^{2}$ Heterobilharzia and Schistosomatium;

${ }^{3}$ S. sinensium, S. japonicum, S. malayensis, S. mekongi; ${ }^{4}$ S. incognitum, S. spindale, S. nasale and S. indicum.

\section{DISCUSSION}

The combination of 3 genes has provided a phylogeny of the Schistosomatidae that is reasonably well resolved at all levels within the tree. The full data set estimated a tree that is unique when compared to the estimates from the individual nuclear ribosomal DNA and mitochondrial cytochrome oxidase I gene trees, but the same broad patterns emerge from each. The main avian clade includes Bilharziella, Trichobilharzia, Dendritobilharzia, and Gigantobilharzia; hereafter referred to as the $B T D G$ clade. Its sister clade includes the mammalian taxa, but also some avian schistosomes. Within this clade, the next group to diverge is less certain, based on nodal support alone. The nuclear ribosomal genes both suggest the mammalian clade (Heterobilharzia + Schistosomatium) (the $H S$ clade) but COI supports the remaining avian genera (Ornithobilharzia + Austrobilharzia). The full analysis reflects the individual ribosomal solutions. Intuitively it might be expected that avian clades gave rise to a monophyletic mammalian clade, with the adoption of a mammalian vertebrate host as a single evolutionary event. Constraint analyses failed to reject either of these two clades as the true sister group to Schistosoma and so phylogenetic analyses were conducted without the use of an outgroup (results not shown) since the poor support for these internal deep nodes may be related to the longbranching outgroup spuriously polarizing these basal taxa (Felsenstein, 1978). However, the unrooted phylograms from all analyses reflected the same patterns in each rooted analysis and the final, full data set indicated a very short branch length between the $B T D G$ and $H S$ clades that, in turn, resulted in relatively low bootstrap support at this critical node. Although support for the relative positions of the $B T D G$ and $H S$ clades remains problematic when assessed in isolation, Bayesian inference strongly recognized Ornithobilharzia + Austrobilharzia as the sister group to the Schistosoma clade, and we consider the full analysis to be the best available estimate. Indeed, the clade is also well supported morphologically (see below). The topology from our combined evidence solution provides the foundation for the following discussion. Specific implications suggested by this new topology are discussed below.

\section{Systematics and taxonomy}

The overall topology of the schistosomatid genera is identical to that presented by Snyder \& Loker (2000), based on partial lsrDNA, except for the position of the root. Snyder \& Loker (2000) resolved Schistosoma + Orientobilharzia as a sister clade to the other schistosomes, but all other relationships are the same. Thus, this study confirms that at least one higher taxonomic group is now clearly challenged. The Schistosomatidae has been subdivided into 4 subfamilies. Leaving aside Griphobilharziinae, for which no samples were obtained, of the others Gigantobilharziinae (Mehra, 1940) as amended by Farley (1971) comprises the genera Dendritobilharzia and Gigantobilharzia and remains a valid taxon in our scheme. Likewise, the Schistosomatinae Stiles \& Hassall, 1926, which includes all remaining taxa other than Bilharziella and Trichobilharzia, also remains monophyletic in this study. However, Bilharziella and Trichobilharzia do not form a monophyletic clade and therefore the subfamily Bilharziellinae Price, 1929 can be rejected. Although Carmichael (1984) listed 2 characters that appeared synapomorphic for the Bilharziellinae, namely the presence of a gynaecophoric canal only surrounding the genital pore (Carmichael's character 4.2), and the male genital pore well behind the acetabulum and caecal reunion (Carmichael's character 20.2), neither Carmichael (1984) nor Morand \& Müller-Graf (2000) favoured the relationship in their final trees. A further consequence of rejecting the Bilharziellinae is that it is not possible to speculate on the position of Filinobilharzia, within which there are only 2 species, 


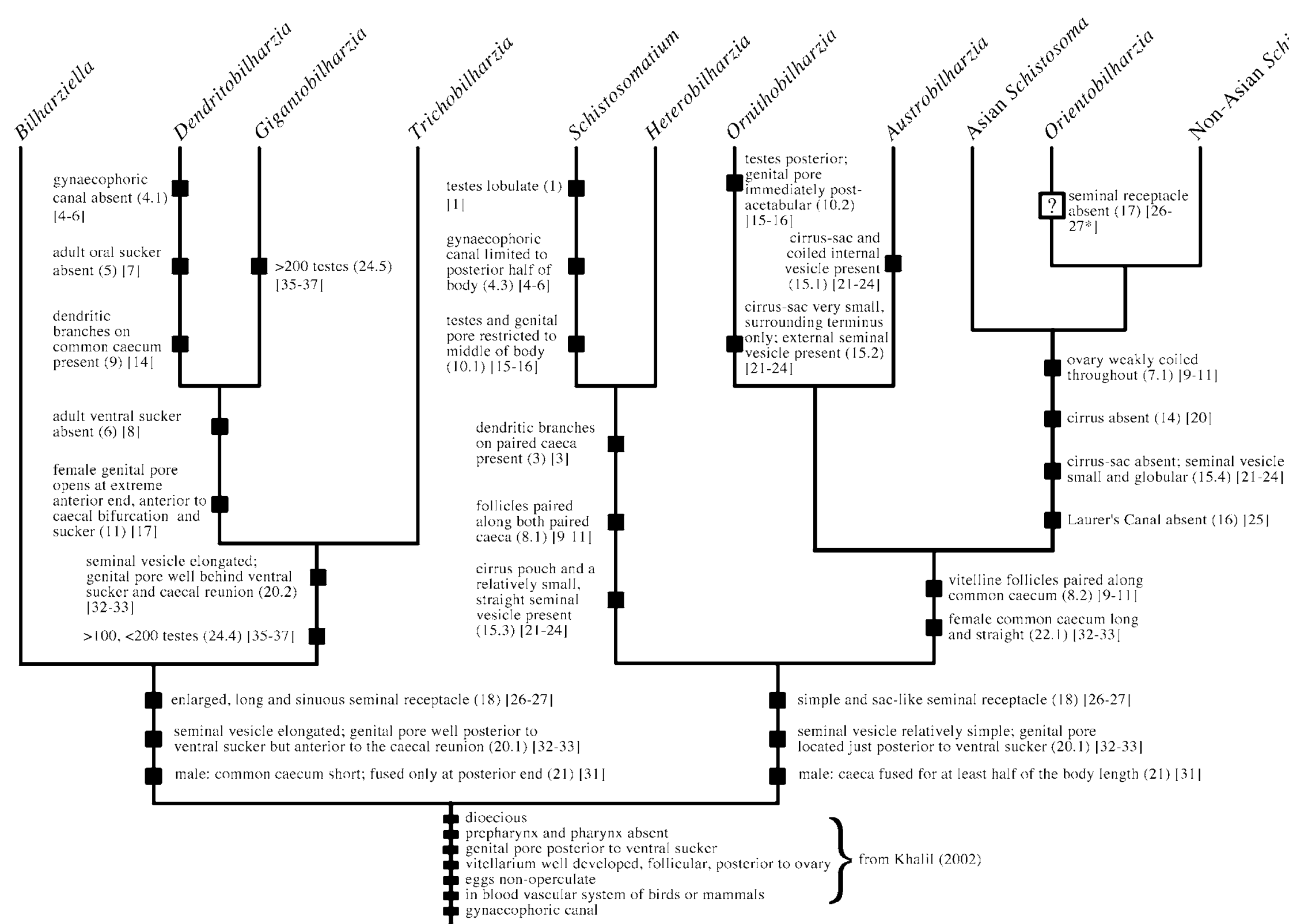

Fig. 4. For legend see facing page. 
beyond suggesting that it will likely fall within the $(B T D G)$ clade.

In order to evaluate our combined evidence tree in the light of morphology, Carmichael's characters (Carmichael, 1984) were mapped on to the tree; the codes used by Morand \& Müller-Graf (2000) are also shown. Highlighting only those characters changing unambiguously (i.e. with a consistency index of 1 ) the character changes are indicated in Fig. 4. In spite of short branch lengths and relatively poor nodal support at the deeper nodes within our tree, a satisfying number of morphological synapomorphies exist at critical nodes throughout the tree. The Schistosomatinae is supported by 3 characters (Carmichael's characters $18,20 \cdot 1,21)$, and the sister group status of (Ornithobilharzia + Austrobilharzia) to Schistosoma is supported by 2 characters concerning the arrangement of the vitelline follicles (8.2) and the length and shape of the female common caecum $(22 \cdot 1)$. Four characters $(7 \cdot 1,14,15 \cdot 4$, and 16) support the reinclusion of Orientobilharzia within Schistosoma, a relationship originally proposed until revised by Dutt \& Srivastava (1961). More recently this relationship was resurrected by Snyder \& Loker (2000) using partial lsrDNA; supported by ITS2 (Zhang et al. 2001) and again partial lsrDNA (Attwood et al. $2002 b$ ); and is confirmed by the present study. Orientobilharzia is traditionally distinguished from Schistosoma by the large number of testes (usually $>50$ ) present in Orientobilharzia (Farley, 1971; Khalil, 2002). Morand \& Müller-Graf (2000) demonstrated that testes number varied widely and not systematically throughout the family, suggesting this is not a good character for phylogenetic purposes. The only autapomorphy for the genus Orientobilharzia (the absence of a seminal receptacle, character 17) may be erroneous, as Carmichael (1984), based on preliminary observations, reported that further study may well reveal the presence of the structure in the 'genus'. It is clear, however, that the genus Orientobilharzia is synonymous with Schistosoma. A revision of the genus Orientobilharzia, ideally supported by molecular evidence, is required.

Within Schistosoma the traditional species groups, based partly on egg morphology, are only marginally compromised by our scheme. The 'japonicum', 'mansoni' and 'haematobium' species groups remain intact but the position of $S$. incognitum renders the 'indicum' group either redundant, or restricted to S.nasale, S. spindale and S. indicum. Egg morphology within the group, with or without $S$. incognitum, is still highly divergent. Of those Schistosoma species not available to us, the position of $S$. hippopotami and $S$. edwardiense remains problematic. Although nominally included in the 'mansoni' species group on the basis of lateral-spined eggs, in the single molecular study that used ITS2 from $S$. hippopotami (Després et al. 1995), it failed to cluster with the $S$. mansoni group. S. hippopotami does show morphological similarities to $S$. incognitum at both egg and adult stages (Thurston, 1963) although the intermediate snail host is not known. Since our results remove $S$. incognitum from the 'indicum' group, it seems likely that these two species could show a close affiliation. No sequence data have ever been obtained from $S$.edwardiense and its position is undetermined, although this species is believed to use Biomphalaria as a snail host (as do S. mansoni and S. rodhaini) (Pitchford \& Visser, 1981).

Attwood et al. (2002a) described S. ovuncatum of northern Thailand as a new species, distinct from $S$ sinensium of Sichuan, China, on the basis of clear morphological difference; the eggs of $S$. ovuncatum bear a small hook-like subterminal spine. Attwood et al. (2002 b) provided partial lsrDNA and mitochondrial $12 \mathrm{~S}$ rDNA sequences for specimens from the type population of $S$. ovuncatum and these indicated that this taxon is the sister species of $S$. sinensium from China $(0 \cdot 8 \%$ of sites were polymorphic at the $28 \mathrm{~S}$ locus and $5 \cdot 3 \%$ at the $12 \mathrm{~S}$ ). Denser sampling within Schistosoma species may indicate high genetic divergence, as found within $S$. sinensium sampled between Thailand and China (Agatsuma et al. 2001) and for S. intercalatum using RAPD data (Kaukas et al. 1994). Divergence levels within $S$. sinensium exceed the divergence between $S$. malayensis and $S$. mekongi and it seems likely that further species will be described. In contrast, sampling of large fragments of mitochondrial DNA within $S$. malayensis and $S$. mekongi populations has shown limited variation (Le, Blair \& McManus, $2002 b$ ).

Our tree suggests one other taxonomically important taxonomic feature. The very close relationship, and almost identical nucleotide sequences between Trichobilharzia ocellata and T. szidati, suggests they may be synonymous. A wider study including denser molecular sampling of populations and a re-evaluation of purported morphological differences will help to confirm this.

\section{Host identity and host associations}

Schistosomatids use snails from across a wide phylogenetic range within the Gastropoda and appear to

Fig. 4. Interpretation of the evolutionary radiation of the Schistosomatidae with the acquisition and loss of key features. Numbers in rounded brackets are the characters from Carmichael (1984), while those in square brackets the numbers assigned for the same characters by Morand \& Müller-Graf (2000). Character 17 of Carmichael (1984), is marked as '?'; although absence of a seminal receptacle is a characteristic of Orientobilharzia, Carmichael (1984), suggested that such a structure may have been visible in one specimen he observed (USNHC \#45820). * - Morand \& Müller-Graf (2000) coded the seminal receptacle using two characters (26 and 27); for Orientobilharzia it was coded as 'simple and sac-like' rather than absent. 
have switched intermediate host across considerable phylogenetic distances (Blair et al. 2001). Our own review of the literature, to assess mollusc families indicated as intermediate hosts to schistosomatids in the wild, is recorded in Fig. 5. Data for individual species used in this study and the genera as a whole are indicated. As a literature review, this figure reflects the completeness and accuracy of the published data. This is not without its difficulties due to the problems in identifying mollusc species and emerging cercariae and it is recognized that the list may include anomalous associations that require at least resampling and at best experimental verification. Our review of this literature suggested that the schistosomatids are generalists in their use of intermediate snail hosts. The same data are presented in Fig. 6 with phylogenies of the molluscs and parasites interlinked by their recorded associations. The basic mollusc phylogeny is adapted from the tree presented by Blair et al. (2001). The interrelationships of the cerithioidean gastropods are adapted from the molecular systematic analysis of Lydeard et al. (2002). Snail host and parasite phylogenies and associations were drawn with the aid of TreeMAP (Page, 1995) in an attempt to identify any patterns of cospeciation. A heuristic search, superimposing the parasite tree on the host tree resolved only 1 cospeciation event and 101 sorting events (see Page, 1994). Intermediate host associations provide little evidence as to the interrelationships of the parasites at the generic level, and it seems likely that there have been several hostswitching events into related taxa. However, the 'tanglegram' indicates the huge variety of snails used by schistosomatids and also indicates how clades within Schistosoma have radiated predominantly within 3 families of snails. The East Asian clade is restricted to the Pomatiopsidae (Gastropoda: Caenogastropoda), while all other species are restricted to the Planorbidae with the exception of S. incognitum and Orientobilharzia. These species both use snails of the Lymnaeidae. Only the other mammalian clade (Heterobilharzia + Schistosomatium) shows a restricted use of snail hosts (Lymnaeidae) whereas it is the avian schistosomes that appear to be transmitted by the greatest diversity of snails. Caenogastropod hosts are only used by the East Asian Schistosoma clade, the (Ornithobilharzia + Austrobilharzia) clade, Gigantobilharzia and 1 species of Trichobilharzia (T. corvi), and again, it is the Schistosoma that are restricted to a single snail family (Pomatiopsidae). Blair (2001) considered pulmonates to be the ancestral hosts for the schistosomatids, with individual host switching events accounting for those using caenogastropod hosts, but the basal position of Austrobilharzia and Ornithobilharzia in their tree meant that they could not be certain. Our tree, with Ornithobilharzia + Austrobilharzia as sister group to the Schistosoma, adds weight to his argument that association with pulmonate host is the pleisiomorphic condition.
Finally, Fig. 5 highlights situations in which schistosomes can become established in new regions. A case in point is the occurrence of an S. haematobium-like species infecting humans in India, which may be transmitted by Ferrissia tenuis, an ancylid snail (Southgate \& Agrawal, 1990). Although this parasite has never been formally identified and other, perhaps more suitable, hosts are present in the reported focus, this unusual occurrence readily demonstrates the ease with which schistosomes can establish themselves when suitable hosts are present.

\section{Geographical distribution and historical biogeography}

As with intermediate host identity, the geographical distribution of taxa studied here, and other members of the genera, is indicated in Fig. 5, again produced from reviewing published work. Not surprisingly, avian schistosomes have a very broad distribution. The most basal avian clade has achieved an almost global distribution with species found in all regions except South America. The wide dispersal of bird parasites is easy to envisage, but the avian schistosomatids' success must also be due to their ability to utilize a variety of molluscan hosts.

With respect to the possible origin and spread of the mammalian genera, Schistosoma and Orientobilharzia (assuming that current distribution is indicative of past events) the Asian origin has been confirmed, with the 'japonicum' group clearly basal to the African and Indian schistosomes. Fig. 7A is a synthesis of Snyder and Loker's hypothesis as reviewed by Morgan et al. (2001). Most interestingly $S$. incognitum, found in India as well as Thailand and Indonesia, diverges early in the phylogeny suggesting that ancestors of the Schistosoma may have entered the Indian subcontinent before Africa; see Fig. 7B for a diagram of our interpretation; Attwood et al. $(2002 b)$ provide finer-scale hypotheses on the movement of Asian Schistosoma into and from the Indian subcontinent. Our results suggest that there has been movement east across Asia giving rise to Orientobilharzia and S. incognitum followed by colonization of Africa from India. There followed a reinvasion of the Indian subcontinent by the ancestor to the 'indicum' species group, which radiated to form $S$. nasale, S. spindale and $S$. indicum, whilst members of the 'haematobium' group continued to radiate within Africa. Such a scenario is dependent upon the position of $S$. nasale within a monophyletic 'indicum' group suggested by the combined evidence. The most recent and most easily dated dispersal ( $~ 500 \mathrm{YA})$ was $S$. mansoni to S. America via the African slave trade (Després et al. 1993), where its subsequent establishment was due to the presence of suitable snail hosts (Campbell et al. 2000; DeJong et al. 2001). The occurrence of 2 lineages within Africa has been recognized for a long time and each lineage has independently given rise to schistosomes that infect 


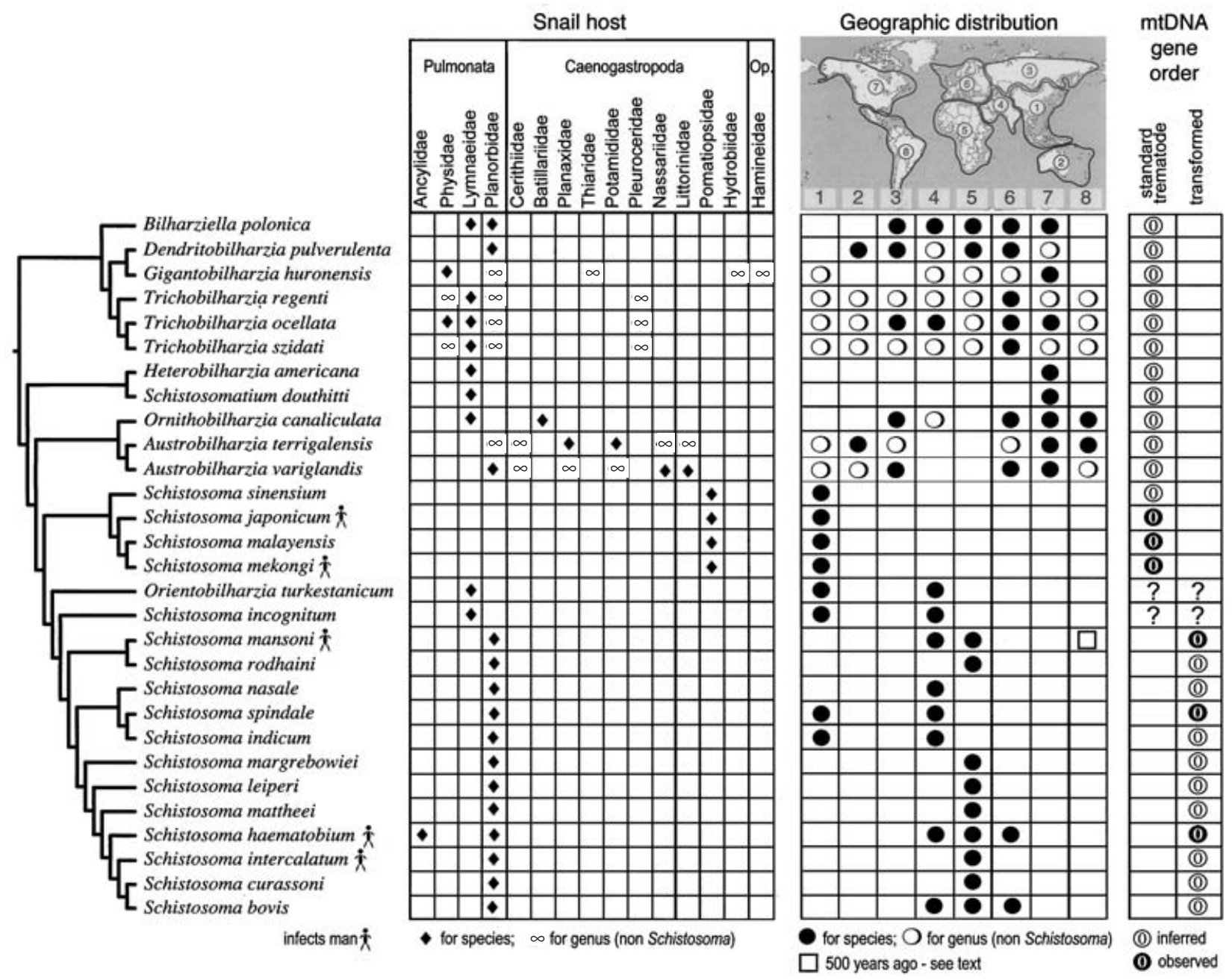

Fig. 5. Intermediate host associations, geographical range and mitochondrial gene order for species studied here, and for all members of each genera (not Schistosoma) as determined from the literature (predominantly the NHM's Host-Parasite Database) or, in the case of mtDNA, inferred from experimental data. Parasites that infect humans are indicated. Op, Opisthobranchia. Snail host associations: $(\bullet)$ indicates that the named species uses that snail family as host; $(\infty)$ indicates that other species within the genus associate with the host family. Geographical distribution: (@) indicates that the named species occupies the specific geographic range indicated; $(\bigcirc)$ indicates that other species within the genus occupy the range indicated.

man, suggesting multiple lateral transfers between hosts, in the case of $S$. mansoni from rodents and for $S$. haematobium and $S$. intercalatum from ungulates (Combes, 1990). A minimum of 3 independent origins of schistosomes in humans can be scored from our phylogeny. To answer the question posed in the title of Zhang et al.'s study (2001), there were at least 1 Asian and 2 African evolutionary origins for human schistosomes. Once associated with humans, the dispersal of schistosomes may have occurred via early humans and their domesticated animals. The fact that schistosomes infecting man seem to be the result of a number of lateral transfers in different lineages indicates that schistosomes were already widespread before the evolution and spread of Homo. This suggests that non-human mammal migration is most likely to be responsible for the continental dispersal of Schistosoma species. Attwood et al. (2002a,b) regarded climate driven dispersal of rodents and other small mammals, rather than human involvement, as a key factor in the early radiation of Schistosoma.

\section{Mitochondrial genomes}

Le et al. (2000) reported a remarkable difference in mitochondrial gene order for S. mansoni when compared to other parasitic platyhelminths, and indeed other Schistosoma species. Comparing sequenced and characterized mitochondrial genomes for 4 species of Schistosoma, 2 other digeneans and 4 cestodes, Le et al. (2001; Le, Blair \& McManus, $2002 a$ ) revealed that $S$. mansoni exhibited a major translocation involving the genes atp6, nad2 and trnaA and a rearrangement of nad 3 and nad1. All the East Asian schistosomes (S. japonicum, S. mekongi and $S$. malayensis) essentially exhibit the same gene 


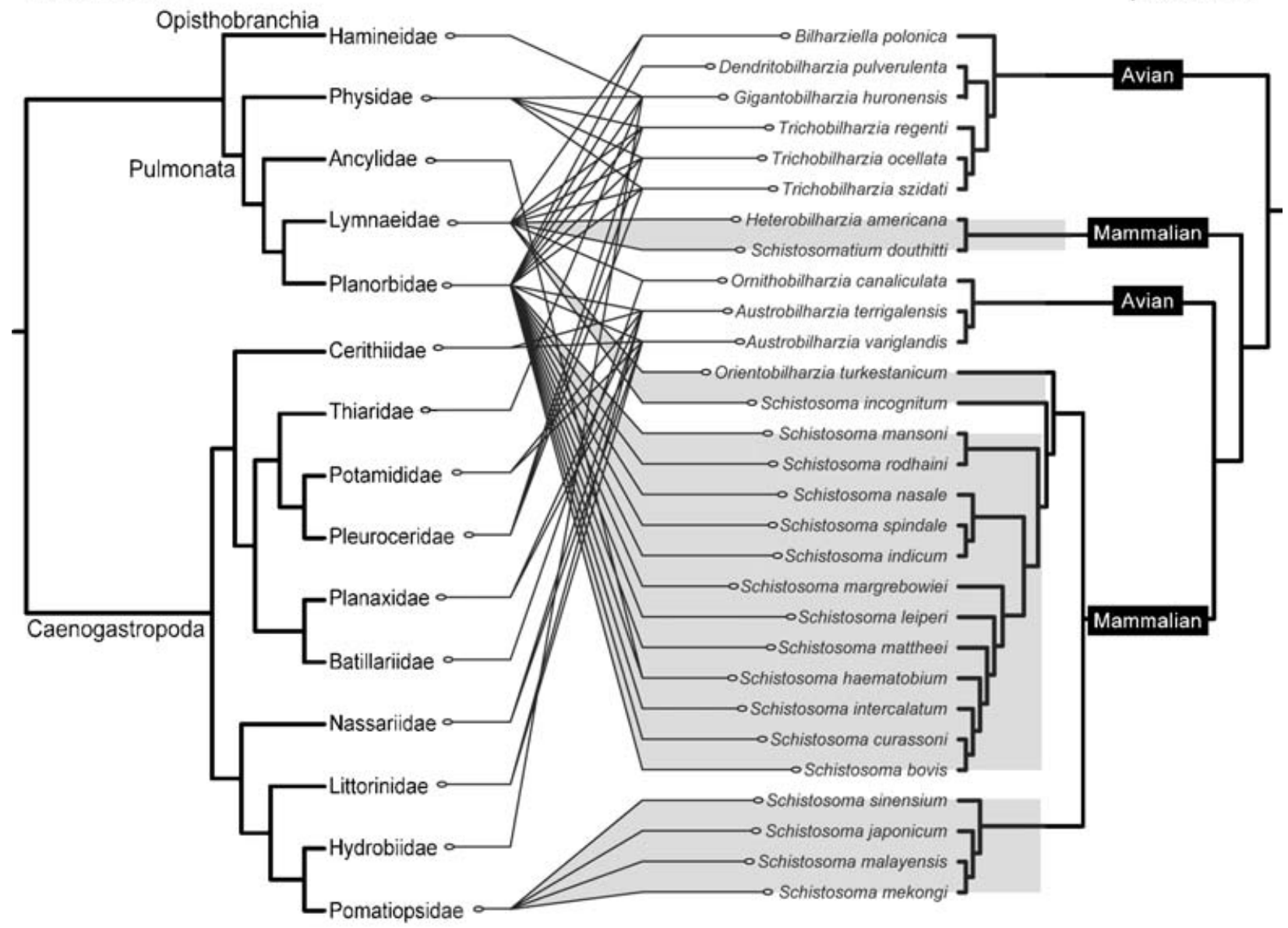

Fig. 6. TreeMap (Page, 1995) plot of snail host and schistosome parasite associations in relation to their respective phylogenies. Avian and mammalian schistosome lineages are indicated. Shaded areas represent snail family associations with the mammalian schistosome clades.

order as other digeneans and cestodes examined to date, suggesting that they have the plesiomorphic condition for the Digenea. It can be inferred therefore, that taxa basal to the East Asian schistosomes also have the plesiomorphic condition and it has recently been confirmed that $S$. spindale and $S$. haematobium have the same gene order as $S$. mansoni (unpublished results). This same gene order is also suggested for 6 other African schistosome species (S.bovis, S.curassoni, S.intercalatum, S.margrebowiei, $S$. mattheei and $S$. rodhaini), based on length and preliminary sequencing of a $2 \cdot 6 \mathrm{~kb}$ fragment of mitochondrial DNA amplified from all 6 species using universal primers (unpublished results). It is therefore inferred that all taxa on our tree from $S$. mansoni to the most derived African taxa have this same gene order. However, the gene order of Orientobilharzia and $S$. incognitum is unknown and since they are likely to hold the key as to when and, based on biogeography, perhaps where the major rearrangement and translocation events occurred, we are currently characterizing these genomes.

The phylogeny presented here, based on 1 mitochondrial protein-coding and 2 nuclear ribosomal
RNA genes, provides a robust assessment of interrelationships within the Schistosomatidae and, in particular, within 18 species of a revised genus Schistosoma that includes Orientobilharzia. Four major clades radiated within the family, reflecting 2 radiations within birds and 2 within mammals. Mammalian schistosomes are relatively restricted in their biogeographical distribution and their use of snail intermediate hosts. Avian schistosomes are more widespread and use a far greater diversity of snail families. There is little evidence for cospeciation between snail families and parasites, although finer scale phylogenies within snail families hosting mammalian schistosomes may subsequently reveal such patterns. We resolved an avian clade, including Austrobilharzia and Ornithobilharzia as sister group to Schistosoma, showing that colonization of mammalian host was not a single event. Within Schistosoma, the East Asian taxa are most basal, confirming an Asian origin for the genus. Subsequent movement of Schistosoma eastwards across Asia resulted in an invasion of Africa, suggested by the position of S. mansoni and $S$. rodhaini, a re-invasion of central Asia and the Indian subcontinent, suggested by the positions of 


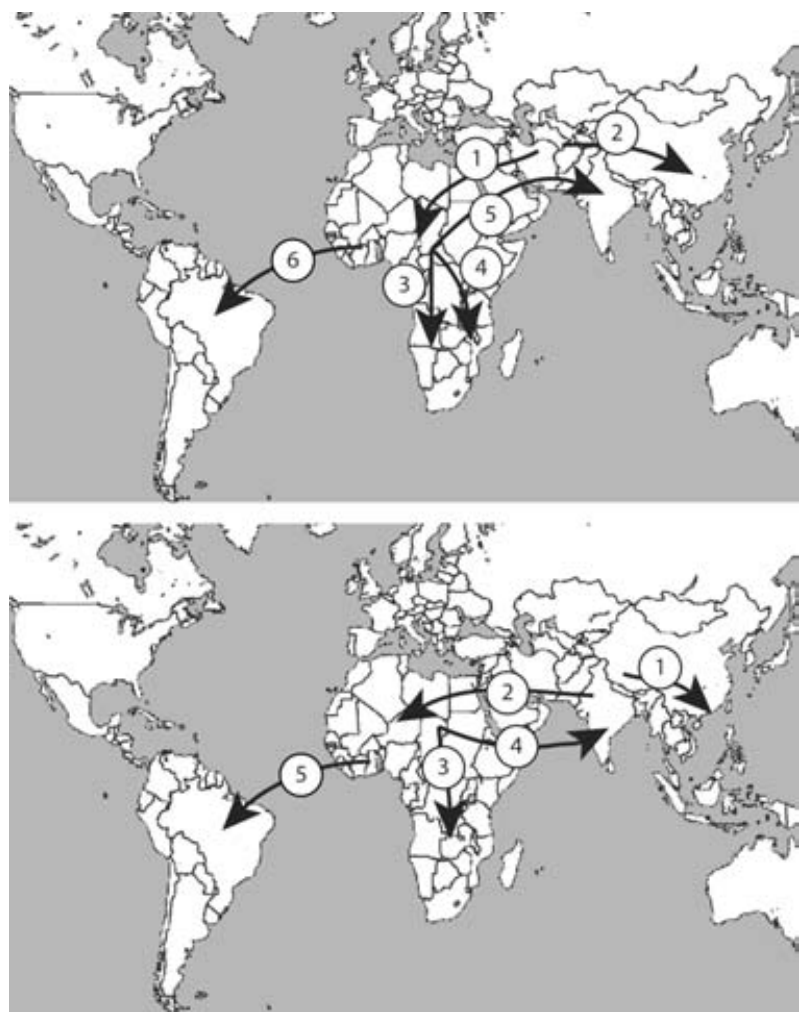

Fig. 7. Hypotheses on the Asian origin and dispersal of Schistosoma. (A) Asian origin (after Morgan et al. 2001; Snyder \& Loker, 2000). (1) The ancestral Asian Schistosoma moves to Africa via widespread mammal migration.

(2) The Schistosoma ancestor remaining in Asia becomes the $S$. japonicum species group. (3 and 4) The African Schistosoma ancestor diverges to form the $S$. mansoni and $S$. haematobium species groups. (5) An $S$. indicum ancestor also diverges from the African ancestor and migrates back to India, probably with early humans and their animals. (6) Schistosoma mansoni disperses to South America via the transport of African slaves. (B) Revised Asian hypothesis (this study). (1) The ancestral Asian Schistosoma radiates into the $S$. japonicum group.

(2) Meanwhile ancestral schistosomes disperse to Africa via India and form the $S$. mansoni group. (3) A subsequent split forms the $S$. haematobium group which radiates through Africa, and (4) the $S$. indicum group which has reinvaded the Indian subcontinent. (5) Schistosoma mansoni disperses to South America via the transport of African slaves.

S. nasale, S. spindale and S. indicum, and an additional radiation of species within Africa among the 'haematobium' species group. Non-human mammal migration is most likely to be responsible for the earlier continental dispersal of Schistosoma species. A suite of morphological characters supports the molecular tree and a number of morphological synapomorphies are recognized for all but a few clades. We reject the subfamily Bilharziellinae, suggest that Trichobilharzia ocellata and T. szidati may be the same species, and advise a revision and renaming of Orientobilharzia to reflect its unquestioned position within the genus Schistosoma. Our tree supports a split in the pattern of mitochondrial genome organization between the East Asian Schistosoma and the more derived taxa, but it remains to be seen whether S. incognitum and/or Orientobilharzia have the plesiomorphic or derived unique patterns of mitochondrial gene arrangement exhibited by $S$. mansoni.

This work was funded by a Wellcome Trust Senior Research Fellowship to D. T.J.L. (043965/Z/95/Z). Additional funding was from the Czech Ministry of Education (Grant No. J13/981131-4) for Petr Horak; from the National Science Foundation (USA) (BIR-9626072) for Scott Snyder, and from Grant-in-Aid for International Scientific Research from the Japanese Ministry of Education $(10041188,13576008)$ for Takeshi Agatsuma. We would like to thank all the many colleagues and fieldworkers who have helped us collect specimens and maintain schistosome life-cycles, especially Mike Anderson, NHM and also M. M. H. Mondal and V. Kitikoon for collecting $S$. indicum and $S$. incognitum respectively. We are grateful to David Gibson, Rod Bray and Eileen Harris for access to the NHM's Host-Parasite Database and help with the systematic literature. Julia Llewellyn-Hughes and Claire Griffin provided expert technical assistance in the operation of the automated DNA sequencers. Fred Naggs and David Reid kindly advised on certain molluscan queries.

\section{REFERENCES}

AGATSUMA, T., IWAGAMi, M., LiU, C. X., RAJAPAKSE, R., MONDAL, M. M. H., KiTiKoOn, V., AMBU, S., AGATSUma, Y., BLAIR, D. \& HIGUCHI, T. (2002). Affinities between Asian non-human Schistosoma species, the S. indicum group, and the African human schistosomes. Fournal of Helminthology 76, 7-19.

agatsuma, T., IWAGAMI, M., LIU, C. X., SAITOH, Y., KAWANAKA, M., UPATHAM, S., QUI, D. \& HIGUCHI, T. (2001). Molecular phylogenetic position of Schistosoma sinensium in the genus Schistosoma. Fournal of Helminthology 75, 215-221.

ATtwood, s. W. (2001). Schistosomiasis in the Mekong region: epidemiology and phylogeography. Advances in Parasitology 50, 87-152.

ATtwood, s. W. \& JOHnston, D. A. (2001). Nucleotide sequence differences reveal genetic variation in Neotricula aperta (Gastropoda : Pomatiopsidae), the snail host of schistosomiasis in the lower Mekong Basin. Biological Fournal of the Linnean Society 73, 23-41. ATtwood, s. W., PANAsoponkul, C., Upatham, E. S., MENG, X. H. \& SOUTHGATE, V. R. (2002a). Schistosoma ovuncatum n. sp. (Digenea: Schistosomatidae) from northwest Thailand and the historical biogeography of Southeast Asian Schistosoma Weinland, 1858. Systematic Parasitology 51, 1-19.

ATTWOOD, S. W., UPATHAM, E. S., MENG, X. H., QIU, D.-C. \& SOUTHGate, V. R. (2002 b). The phylogeography of Asian Schistosoma (Trematoda: Schistosomatidae). Parasitology 125, 99-112.

BARKER, S. C. \& BLAIR, D. (1996). Molecular phylogeny of Schistosoma species supports traditional groupings within the genus. Fournal of Parasitology 82, 292-298.

BARRAL, V., THIS, P., IMBERT-ESTABLET, D., COMBES, C. \& DELSENY, M. (1993). Genetic variability and evolution of the Schistosoma genome analysed by using random 
amplified polymorphic DNA markers. Molecular and Biochemical Parasitology 59, 211-222.

BLAIR, D., DAVIS, G. M. \& WU, B. (2001). Evolutionary relationships between trematodes and snails emphasizing schistosomes and paragonimids. Parasitology 123, S229-S243.

BLAIR, D., VAN HERWERdEN, L., HiRAI, H., TAGUChI, T., HABE, S., hirata, M., LAI, K., UPATHAM, S. \& AGATSUMA, T. (1997). Relationships between Schistosoma malayensis and other Asian schistosomes reduced from DNA sequences. Molecular and Biochemical Parasitology 85, 259-263.

BOWLES, J., BLAIR, D. \& McMANUS, D. P. (1995). A molecular phylogeny of the human schistosomes. Molecular phylogenetics and Evolution 4, 103-109.

CAMPBELl, G., JONES, C. S., LOCKYER, A. E., HUGHES, S., BROWN, D., NOBLE, L. R. \& ROLLINSON, D. (2000). Molecular evidence supports an African affinity of the Neotropical freshwater gastropod, Biomphalaria glabrata, Say 1818, an intermediate host for Schistosoma mansoni. Proceedings of the Royal Society of London, $B$ 267, 2351-2358.

Carmichael, A. C. (1984). Phylogeny and historical biogeography of the Schistosomatidae. Ph.D. thesis, Michigan State University, Michigan.

COMBEs, c. (1990). Where do human schistosomes come from? An evolutionary approach. Trends in Ecology and Evolution 5, 334-337.

CRIBB, T. H., BRAY, R. A., LITTLEWOOD, D. T. J., PICHELIN, S. P. \& Herniou, E. A. (2001). The Digenea. In

Interrelationships of the Platyhelminthes (ed. Littlewood, D. T. J. \& Bray, R. A.), pp. 168-185. Taylor \& Francis, London.

CUNNinghaM, C. w. (1997). Can three incongruence tests predict when data should be combined? Molecular Biology and Evolution 14, 733-740.

CUTLER, D. J. (2000). Estimating divergence times in the presence of an overdispersed molecular clock. Molecular Biology and Evolution 17, 1647-1660.

DAvis, G. M. (1980). Snail hosts of Asian Schistosoma infecting man: evolution and coevolution. In The Mekong Schistosome (ed. Bruce, J. I., Sornmani, S., Asch, H. L. \& Crawford, K. A.), pp. 195-238.

Malacological Review Suppl. 2, Whitmore Lake, Michigan.

DE BOnt, J. \& VERCRuYsse, J. (1997). The epidemiology and control of cattle schistosomiasis. Parasitology Today 13, $255-262$.

DEJONG, R. J., MORgan, J.A. T., PARAENSE, W. L., POINTIER, J. P., AMARISTA, M., AYEH-KUMI, P. F. K., BABIKER, A., BARbosa, C. S., BREMOND, P., CANESE, A. P., DE SOUZA, C. P., DOMingueZ, C., FILE, S., GUTIERREZ, A., INCANI, R. N., KAWANO, T., KAZIBWE, F., KPIKPI, J., LWAMBO, N. J. S., MIMPFOUNDI, R., NJIOKOU, F., PODA, J. N., SENE, M., velasqueZ, L. E., YONG, M., ADEMA, C. M., HOFKIN, B. V., MKOJI, G. M. \& LOKER, E. S. (2001). Evolutionary relationships and biogeography of Biomphalaria (Gastropoda: Planorbidae) with implications regarding its role as host of the human bloodfluke, Schistosoma mansoni. Molecular Biology and Evolution 18, 2225-2239.

DESPRÉs, L., IMBERT-ESTABLET, D., COMBES, C. \& BONHOMME, F. (1992). Molecular evidence linking hominid evolution to recent radiation of schistosomes (Platyhelminthes:
Trematoda). Molecular Phylogenetics and Evolution 1, 295-304.

DESPRÉS, L., IMBERT-ESTABLET, D. \& MONNEROT, M. (1993). Molecular characterization of mitochondrial DNA provides evidence for the recent introduction of Schistosoma mansoni into America. Molecular and Biochemical Parasitology 60, 221-230.

DESPRÉs, L., KRUGER, F. J., IMBERT-ESTABLET, D. \& ADAMSON, M. L. (1995). ITS2 ribosomal RNA indicates Schistosoma hippopotami is a distinct species. International Fournal for Parasitology 25, 1509-1514.

DOWTON, M. \& AUSTIN, A. D. (2002). Increased congruence does not necessarily indicate increased phylogenetic accuracy - the behavior of the incongruence length difference test in mixed-model analyses. Systematic Biology 51, 19-31.

DUTT, S. C. \& SRIVASTAVA, H. D. (1961). A revision of the genus Ornithobilharzia Odhner (1912) with the creation of two genera Orientobilharzia Dutt and Srivastava (1955) and Sinobilharzia Dutt and Srivastava (1955) (Trematoda: Schistosomatidae). Indian Fournal of Helminthology 13, 61-73.

DVORAK, J., VANACOVA, S., HAMPL, V., FLEGR, J. \& HORAK, P. (2002). Comparison of European Trichobilharzia species based on ITS1 and ITS2 sequences. Parasitology 124, 307-313.

FARLEY, J. (1971). A review of the family Schistosomatidae: excluding the genus Schistosoma from mammals. Fournal of Helminthology 45, 289-320.

FARris, J. S., KAllersjo, M., KLUGE, A. G. \& BUlt, C. (1995). Constructing a significance test for incongruence. Systematic Biology 44, 570-572.

FELsenstein, J. (1978). Cases in which parsimony or compatibility methods will be positively misleading. Systematic Zoology 27, 401-410.

GIBSON, D. I. \& BRAY, R. A. (1994). The evolutionary expansion and host-parasite relationships of the Digenea. International Fournal for Parasitology 24, 1213-1226. GIBSON, D. I., JONES, A. \& BRAY, R. A. (2002). Keys to the Trematoda. Vol. 1. CABI Publishing, Wallingford. HORAK, P. \& KOLAROVA, L. (2001). Bird schistosomes - do they die in mammalian skin? Trends in Parasitology 17, 66-69.

HORAK, P., Kolarova, L. \& ADEMA, C. M. (2002). Biology of the schistosome genus Trichobilharzia. Advances in Parasitology 52, 155-233.

HuELSENBECK, J. P. (2000). MrBayes: Bayesian inference of phylogeny. Department of Biology, University of Rochester.

HUElSENBECK, J. P., BULl, J. J. \& CUNNINGHAM, C. W. (1996). Combining data in phylogenetic analysis. Trends in Ecology and Evolution 11, 152-158.

JeAnMougin, F., Thompson, J. D., GOUY, M., Higgins, D. G. \& GIBSON, T. J. (1998). Multiple sequence alignment with Clustal X. Trends in Biochemical Sciences 23, 403-405.

JOHNSTON, D. A., KANE, R. A. \& ROLLINSON, D. (1993). Small subunit (18S) ribosomal RNA gene divergence in the genus Schistosoma. Parasitology 107, 147-156.

KaUKas, A., Dias NETO, E., Simpson, A. J. G., SOUthGate, V. R. \& ROLLINSON, D. (1994). A phylogenetic analysis of Schistosoma haematobium group species based on randomly amplified polymorphic DNA. International Fournal for Parasitology 24, 285-290. 
KHALIL, L. F. (2002). Family Schistosomatidae Stiles \& Hassall, 1898. In Keys to the Trematoda (ed. Gibson, D. I., Jones, A. \& Bray, R. A.), pp. 419-432. CABI Publishing, Wallingford, UK.

LE ROUX, P. L. (1958). The validity of Schistosoma capense (Harley, 1864) amended as a species. Transactions of the Royal Society of Tropical Medicine and Hygiene 52, 12-14.

LE, T. H., BlAir, D., AGATSUMA, T., HUMAIR, P. F., CAMPBELL, N. J. H., IWAGAMI, M., LITTLEWOOD, D. T. J., PEACOCK, B., JOHNSTON, D. A., BARTLEY, J., ROLLINSON, D., HERNIOU, E. A., ZARLENGA, D. S. \& McMANUS, D. P. (2000).

Phylogenies inferred from mitochondrial gene orders - a cautionary tale from the parasitic flatworms. Molecular Biology and Evolution 17, 1123-1125.

LE, T. H., BLAIR, D. \& McMAnUs, D. P. (2001). Complete DNA sequence and gene organization of the mitochondrial genome of the liverfluke, Fasciola hepatica $\mathrm{L}$. (Platyhelminthes: Trematoda). Parasitology 123, 609-621.

LE, T. H., BLAIR, D. \& McMAnUs, D. P. (2002a). Mitochondrial genomes of parasitic flatworms. Trends in Parasitology 18, 206-213.

LE, T. H., BLAIR, D. \& McMANUS, D. P. (2002b). Revisiting the question of limited genetic variation within Schistosoma japonicum. Annals of Tropical Medicine and Parasitology 96, 155-164.

LEE, M. S. Y. (2001). Uninformative characters and apparent conflict between molecules and morphology. Molecular Biology and Evolution 18, 676-680.

LitTlewood, D. T. J. \& JOHnston, D. A. (1995). Molecular phylogenetics of the four Schistosoma species groups determined with partial $28 \mathrm{~S}$ ribosomal RNA gene sequences. Parasitology 111, 167-175.

LITTLEWOOD, D. T. J., ROHDE, K., BRAY, R. A. \& HERNIOU, E. A. (1999). Phylogeny of the Platyhelminthes and the evolution of parasitism. Biological Fournal of the Linnean Society 68, 257-287.

LOCKYER, A. E., OLSON, P. D. \& LITTLEWOOD, D. T. J. (2003). Utility of complete large and small subunit rRNA genes in resolving the phylogeny of the Platyhelminthes: implications and a review of the cercomer theory. Biological Fournal of the Linnean Society 78, 155-173.

LYDEARD, C., HOLZNAGEL, W. E., GLAUBRECHT, M. \& PONDER, W. F. (2002). Molecular phylogeny of a circum-global, diverse gastropod superfamily (Carithioidea: Mollusca: Caenogastropoda): pushing the deepest phylogenetic limits of mitochondrial LSU rDNA sequences. Molecular Phylogenetics and Evolution 22, 399-406.

MADDISON, W. P. \& MADDISON, D. R. (2000). MacClade. Version 4. Sinauer Associates, New York.

MALlatT, J. \& WINChell, C. J. (2002). Testing the new animal phylogeny: first use of combined large-subunit and small-subunit rRNA gene sequences to classify the Protostomes. Molecular Biology and Evolution 19, 289-301.

MEdina, M., COlLiNs, A. G., Silberman, J. D. \& SOGIN, M. L. (2001). Evaluating hypotheses of basal animal phylogeny using complete sequences of large and small subunit rRNA. Proceedings of the National Academy of Sciences, USA 98, 9707-9712.

MORAND, S. \& MÜLLER-GRAF, C. D. M. (2000). Muscles or testes? Comparative evidence for sexual competition among dioecious blood parasites (Schistosomatidae) of vertebrates. Parasitology 120, 45-56.

MORGAN, J. A. T., DE JONG, R. J., SNYDER, S. D., MKOJI, G. M. \& LOKER, E. S. (2001). Schistosoma mansoni and Biomphalaria: past history and future trends. Parasitology 123, S211-S228.

OLson, P. D. \& LiTTLEWood, D. T. J. (2002). Phylogenetics of the Monogenea - evidence from a medley of molecules. International Fournal for Parasitology 32, 233-244.

OLSON, P. D., LITTLEWOOD, D. T. J., BRAY, R. A. \& MARIAUX, J. (2001). Interrelationships and evolution of the tapeworms (Platyhelminthes: Cestoda). Molecular Phylogenetics and Evolution 19, 443-467.

PAGE, R. D. M. (1994). Maps between trees and cladistic analysis of historical associations among genes, organisms, and areas. Systematic Biology 43, $58-77$.

Page, R. D. M. (1995). TreeMap. Ver. 1.0. Division of Environmental and Evolutionary Biology, University of Glasgow.

PITCHFORD, R. J. \& VISSER, P. S. (1981). Schistosoma, Weinland, 1858 from Hippopotamus amphibious, Linnaeus 1758 in the Kruger National Park. Onderstepoort Fournal of Veterinary Research 49, 233-235.

PLATT, T. R., BLAIR, D., PURDIE, J. \& MELVILlE, L. (1991). Griphobilharzia amoena n. gen, n. sp. (Digenea, Schistosomatidae), a parasite of the freshwater crocodile Crocodylus johnstoni (Reptilia, Crocodylia) from Australia, with the erection of a new subfamily, Griphobilharziinae. Fournal of Parasitology 77, 65-68.

PLATT, T. R. \& BROOKS, D. R. (1997). Evolution of the schistosomes (Digenea: Schistosomatoidea): the origin of dioecy and colonization of the venous system. Fournal of Parasitology 83, 1035-1044.

POSADA, D. \& CRANDALL, K. A. (1998). Modeltest: testing the model of DNA substitution. Bioinformatics 14, 817-818.

RAMbaUT, A. (2000). Estimating the rate of molecular evolution: incorporating non-contemporaneous sequences into maximum likelihood phylogenies. Bioinformatics 16, 395-399.

ROLLINSON, D., KAUKAS, A., JOHNSTON, D. A., SIMPSON, A. J. G. \& TANAKA, M. (1997). Some molecular insights into schistosome evolution. International fournal for Parasitology 27, 11-28.

ROLlinson, D. \& SOUTHGate, v. R. (1987). The genus Schistosoma: a taxonomic appraisal. In The Biology of Schistosomes. From Genes to Latrines (ed. Rollinson, D. \& Simpson, A. J. G.), pp. 1-49. Academic Press, London.

SAMBROOK, J., FRITSCH, E. F. \& MANIATIS, T. (1989). Molecular Cloning. A Laboratory Manual. Cold Spring Harbour Laboratory Press, Cold Spring Harbour. Shimodaira, H. \& HasegaWa, M. (1999). Multiple comparisons of log-likelihoods with applications to phylogenetic inference. Molecular Biology and Evolution 16, 1114-1116.

SNYDER, S. D. \& LOKER, E. S. (2000). Evolutionary relationships among the Schistosomatidae (Platyhelminthes: Digenea) and an Asian origin for Schistosoma. Fournal of Parasitology 86, 283-288. 
SoUthGate, v. R. \& AGRawal, M. C. (1990). Human schistosomiasis in India? Parasitology Today 6, 166-168.

SWOFford, D. L. (2002). PAUP*. Phylogenetic Analysis Using Parsimony *and Other Methods, Ver. 4. Sinauer Associates, New York.

Thurston, J. P. (1963). Schistosomes from Hippopotamus amphibius L. I The morphology of Schistosoma hippopotami sp. nov. Parasitology 53, 49-54.
WORLD HEALTH ORGANIZATION (2001). The World Health Report 2001 : Mental Health: New Understanding, New Hope. World Health Organization, Geneva.

ZHANG, G. J., VERNEAU, O., QIU, C. P., JOURDANE, J. \& XIA, M. Y. (2001). An African or Asian evolutionary origin for human schistosomes? Comptes Rendus de l'Academie des Sciences Serie III. Sciences de la vie 324, 1001-1010. 